\title{
EXACT ANALYSIS OF A NONLINEAR PARTIAL DIFFERENTIAL EQUATION OF GAS DYNAMICS
}

\author{
BY \\ P. L. SACHDEV (Indian Institute of Science, Bangalore, India), \\ S. DOWERAH ${ }^{\dagger}$, \\ B. MAYIL VAGANAN (Indian Institute of Science, Bangalore, India), \\ AND \\ VARUGHESE PHILIP (Regional Engineering College, Calicut, India)
}

\begin{abstract}
A new second-order nonlinear partial differential equation is derived from one-dimensional unsteady non-isentropic gas-dynamic equations through the introduction of three "potential" functions. Appropriate boundary conditions at the shock and at the piston in terms of the new functions are obtained. The nonlinear partial differential equation is analysed in great detail. Intermediate integrals and generalized Riemann invariants are discovered. Using the classical Lie group method, the direct similarity method due to Clarkson and Kruskal (1989), and equation-splitting etc., large families of new solutions are found. The direct similarity method is found to yield the most general results. Solutions with shocks (both finite and strong) are constructed to illustrate the applicability of the solutions.
\end{abstract}

1. Introduction. The system of nonlinear partial differential equations describing plane gas flows have been studied for a long time (see Courant and Friedrich [1]). A typical physical problem is to determine the trajectory of a shock produced by a given piston motion. The flow between the piston and the shock is in general non-isentropic. Some of the earlier analytic studies on this topic used notions of self-similarity to reduce the problem to that of a system of nonlinear ordinary differential equations subject to boundary conditions at the shock and at the piston (see Sedov [2], Keller [3], and McVittie [4]). This approach places severe restrictions on the form of the solutions. From all the previous work it would appear that new exact solutions are difficult to find, at least in the physical plane; one must seek other forms of the basic equations that would allow the boundary conditions, both at the piston and at the shock, to be conveniently satisfied.

An early attempt in this direction is due to Martin [5] and Ludford and Martin [6], who transformed the system of equations for unsteady, non-isentropic adiabatic flows with an

Received August 12, 1993.

1991 Mathematics Subject Classification. Primary 76N15, 76N20.

${ }^{\dagger}$ Since deceased.

(C)1997 Brown University 
arbitrary equation of state into a Monge-Ampère equation. In the latter study, Ludford and Martin [6] found intermediate integrals both for isentropic and non-isentropic flows and interpreted them as simple waves and generalized simple waves. A good qualitative study of the mathematical solutions was given; however, no physical solutions, in particular none with shocks, were found.

In another formulation, Steketee [7], [8] started with equations in Lagrangian coordinates. He introduced functions that are similar to potentials and stream functions, as well as their Legendre transformations. These arise naturally from the divergence form of the Lagrangian equations. The forms obtained by Steketee [8] included those of Ludford and Martin [6] and Smith [9] as special cases. He obtained generalized Riemann invariants for the Ludford-Martin-Stanyukovich gas and illustrated one of the solutions as a generalized simple wave. The second-order nonlinear partial differential equation (see Steketee [8]), equivalent to the first-order system, has a rather simple structure so that there exist solutions that are homogeneous in the Lagrangian coordinate and time and have nice properties.

Ardavan-Rhad [10], in his study of the decay of a plane shock due to interaction with a centered simple wave, derived from the basic equations of gas dynamics a second-order nonlinear partial differential equation with particle velocity as dependent variable and sound speed and entropy as independent variables. However, the intermediate integral for this partial differential equation found by Ardavan-Rhad [10] involved only one arbitrary function and so could not yield the exact solution of the interaction problem. The partial differential equation derived by Ardavan-Rhad [10] is quite complicated and has yet not been treated in any generality.

Ustinov [11] introduced a very interesting system of coordinates such that the shock trajectory was given by a constant value of one of the coordinates while the piston path and the particle trajectories were marked by constant values of the other coordinate. He found a special class of solutions which described gas flows, headed by a shock into a non-uniform undisturbed medium. These flows are produced by a certain piston motion. Some flows without shocks were also found by allowing the shock strength to tend to zero. This work was generalized by Sachdev and Reddy [12] who, using the method of infinitesimal transformations, found several classes of solutions describing flows with shocks, which either decay or grow in a finite or infinite time, depending on the density distribution in the ambient medium; the shock strength, as measured by the pressure ratio, however, remained constant. Sachdev and Gupta [13] dispensed with the similarity assumption in the work of Sachdev and Reddy [12] and significantly enlarged the scope of exact solutions. By adopting series solutions, the solutions obtained by Sachdev and Reddy [12] were embedded in a larger family of solutions.

Ustinov [14], [15], [16] introduced yet another coordinate system, which arises from a different set of conservation laws of the one-dimensional gas-dynamic equations. It turns out that an equivalent total differential form gives rise to a second-order nonlinear partial differential equation (see Eq. (2.20)), which enjoys considerable symmetries and admits much analysis. While the dependent variable is one of the "potential" functions, it does not have a simple physical interpretation; however, the physical variables such as velocity and sound speed can be derived from it in a simple way. One of the independent variables, 
equated to a constant, gives the streamlines while the other is again hard to interpret; for strong shocks this second independent variable is zero along the shock trajectory. Thus, the solutions of (2.20) express relations between three "potential" functions.

The purpose of the present work is to demonstrate that (2.20) admits a considerable analysis. First, it is possible to find some intermediate integrals in a systematic manner; these integrals, in turn, lead to explicit solutions. There are also exact solutions, which are quadratics in one of the independent variables with the coefficients depending on the other independent variable. This form of the solutions arises naturally from consideration of the problem of a short intense impact of a piston moving under gas pressure. The invariance of (2.20) under the group of infinitesimal transformations reveals several classes of similarity solutions. Many of the solutions can be interpreted as flows produced by piston motions and headed by strong shocks; there are also solutions that describe shocks of finite strength. We apply the direct similarity method (see Clarkson and Kruskal [17]) to $(2.20)$ to identify the most general similarity forms of solutions of $(2.20)$. These similarity forms involve five functions of which four are governed by a system of coupled nonlinear ordinary differential equations while the fifth, the similarity function, is given by a second-order nonlinear ordinary differential equation. It does not seem possible to solve this system generally. However, some special solutions are obtained and shown to contain those obtained by the other methods including the group-theoretic method [18].

The scheme of the present paper is as follows. The governing equations, boundary conditions, and their transformations are given in Sec. 2. Intermediate integrals and explicit solutions from them are found in Sec. 3. In Sec. 4 we obtain similarity solutions using the group-theoretic method; in particular, we construct solutions that describe flows with strong shocks or shocks of finite strength. In Sec. 5 more general similarity solutions are obtained using the direct similarity method. In Sec. 6 we directly seek solutions that are quadratic in one of the independent variables with coefficients depending on the other. Section 7 gives solutions obtained by splitting (2.20). The results and conclusions of the present study are contained in Sec. 8 .

\section{The governing equations, boundary conditions, and their transforma-} tions. The equations governing plane, adiabatic flows of an ideal gas are

$$
\begin{aligned}
\frac{\partial \rho}{\partial t}+\frac{\partial(\rho u)}{\partial r} & =0 \\
\frac{\partial u}{\partial t}+u \frac{\partial u}{\partial r}+\frac{1}{\rho} \frac{\partial p}{\partial r} & =0 \\
\frac{\partial\left(p \rho^{-\gamma}\right)}{\partial t}+u \frac{\partial\left(p \rho^{-\gamma}\right)}{\partial r} & =0
\end{aligned}
$$

where $\rho, u$, and $p$ are density, velocity, and pressure, respectively; $r$ is the spatial coordinate and $t$ is time. The constant $\gamma$ is the ratio of specific heats. It is possible to manipulate Eqs. (2.1)-(2.3) so that, apart from (2.1), we have the conservation laws

$$
\begin{aligned}
\frac{\partial}{\partial t}(\rho u)+\frac{\partial}{\partial r}\left(p+\rho u^{2}\right) & =0 \\
\frac{\partial}{\partial t}\left(\frac{1}{\gamma-1} p+\frac{1}{2} \rho u^{2}\right)+\frac{\partial}{\partial r}\left(\frac{\gamma}{\gamma-1} p u+\frac{1}{2} \rho u^{3}\right) & =0 .
\end{aligned}
$$


Equations (2.1) and (2.4) are equivalent to the differential relations

$$
d \tau=\rho d r-\rho u d t=m d y \quad \text { and } \quad d \xi=\rho u d r-\left(p+\rho u^{2}\right) d t=-m a_{0} d x
$$

where $m$ and $a_{0}$ are positive constants, having dimensions $M L^{-2}$ and $L T^{-2}$, respectively. Using (2.6) and the equation of state

$$
f(\tau)=\frac{p^{1 / \gamma}}{(\gamma-1) \rho}
$$

we replace Eq. (2.5) by the total differential

$$
d \eta=u d \xi+\left(f v-\frac{1}{2} u^{2}\right) d \tau=m a_{0}^{2} d z, \quad v=p^{(\gamma-1) / \gamma} .
$$

It is evident from (2.6) and (2.8) that $x, y$, and $z$ are dimensionless variables. Now we proceed to obtain a partial differential equation governing the function $z=z(x, y)$. Equation (2.8) implies the relations

$$
u=-a_{0} \frac{\partial z}{\partial x} \quad \text { and } \quad a_{0}^{2} \frac{\partial z}{\partial y}=\frac{1}{\gamma-1} \frac{p}{\rho}-\frac{1}{2} u^{2}
$$

which, after eliminating $u$, give

$$
\frac{p}{\rho}=a_{0}^{2}(\gamma-1)\left[\frac{\partial z}{\partial y}+\frac{1}{2}\left(\frac{\partial z}{\partial x}\right)^{2}\right] .
$$

Differentiating (2.10) with respect to $x$ and $y$, respectively, we obtain

$$
\begin{aligned}
& \frac{1}{\rho} \frac{\partial p}{\partial x}-\frac{p}{\rho^{2}} \frac{\partial \rho}{\partial x}=a_{0}^{2}(\gamma-1)\left(\frac{\partial^{2} z}{\partial x \partial y}+\frac{\partial z}{\partial x} \frac{\partial^{2} z}{\partial x^{2}}\right) \\
& \frac{1}{\rho} \frac{\partial p}{\partial y}-\frac{p}{\rho^{2}} \frac{\partial \rho}{\partial y}=a_{0}^{2}(\gamma-1)\left(\frac{\partial^{2} z}{\partial y^{2}}+\frac{\partial z}{\partial x} \frac{\partial^{2} z}{\partial x \partial y}\right)
\end{aligned}
$$

Differentiation of the equation of state (2.7) with respect to $x$ and $y$, respectively, yields

$$
\begin{aligned}
& \frac{1}{\rho} \frac{\partial \rho}{\partial x}=\frac{1}{\gamma p} \frac{\partial p}{\partial x} \\
& \frac{1}{\rho} \frac{\partial \rho}{\partial y}=\frac{1}{\gamma p} \frac{\partial p}{\partial y}-\frac{m}{f} \frac{d f}{d \tau}
\end{aligned}
$$

On inserting (2.13) into (2.11) and (2.14) into (2.12), we get

$$
\begin{aligned}
& \frac{1}{\rho} \frac{\partial p}{\partial x}=\gamma a_{0}^{2}\left(\frac{\partial^{2} z}{\partial x \partial y}+\frac{\partial z}{\partial x} \frac{\partial^{2} z}{\partial x^{2}}\right) \\
& \frac{1}{\rho} \frac{\partial p}{\partial y}=\gamma a_{0}^{2}\left(\frac{\partial^{2} z}{\partial y^{2}}+\frac{\partial z}{\partial x} \frac{\partial^{2} z}{\partial x \partial y}\right)-\frac{m \gamma}{\gamma-1} \frac{1}{f} \frac{d f}{d \tau} \frac{p}{\rho}
\end{aligned}
$$


Solving Eqs. (2.6) for $d t$, we have

$$
d t=\frac{m a_{0}}{p} d x+\frac{m u}{p} d y
$$

so that

$$
a_{0} \frac{\partial p}{\partial y}=u \frac{\partial p}{\partial x}-p \frac{\partial u}{\partial x}
$$

On using the first of (2.9), (2.18) becomes

$$
\frac{\partial p}{\partial y}+\frac{\partial z}{\partial x} \frac{\partial p}{\partial x}-\frac{\partial^{2} z}{\partial x^{2}} p=0
$$

Substituting (2.10), (2.15), and (2.16) into (2.19), we arrive at a second-order nonlinear hyperbolic partial differential equation

$$
\begin{aligned}
& \frac{\partial^{2} z}{\partial y^{2}}+2 \frac{\partial z}{\partial x} \frac{\partial^{2} z}{\partial x \partial y}+\left[\frac{\gamma+1}{2 \gamma}\left(\frac{\partial z}{\partial x}\right)^{2}-\frac{\gamma-1}{\gamma} \frac{\partial z}{\partial y}\right] \frac{\partial^{2} z}{\partial x^{2}} \\
&-\phi(y)\left[\frac{\partial z}{\partial y}+\frac{1}{2}\left(\frac{\partial z}{\partial x}\right)^{2}\right]=0
\end{aligned}
$$

where $(m / f)(d f / d \tau)=\phi(y)$.

The Rankine-Hugoniot relations for a plane shock are

$$
\begin{aligned}
& u=\frac{2 U}{\gamma+1}-\frac{2 \gamma p_{0}}{(\gamma+1) \rho_{0} U} \\
& p=\frac{2 \rho_{0} U^{2}}{\gamma+1}-\frac{\gamma-1}{(\gamma+1)} p_{0} \\
& \rho=\frac{(\gamma+1) \rho_{0}^{2} U^{2}}{2 \gamma p_{0}+(\gamma-1) \rho_{0} U^{2}}
\end{aligned}
$$

where $U$ is the shock velocity. The quantities with zero subscript pertain to ambient conditions ahead of the shock. The shock locus is given by

$$
\frac{d r}{d t}=U
$$

The shock conditions (2.21)-(2.23) may be transformed to the $x, y, z$ variables as follows: We assume that the shock locus in the $(x, y)$-plane is given by $y=y_{0}(x)$. On inserting (2.21)-(2.24), the differential relations (2.6) and (2.8) yield

$$
\begin{aligned}
U \rho_{0} d t & =m d y_{0}, \\
p_{0} d t & =m a_{0} d x \\
\frac{p_{0} U}{\gamma-1} d t & =m a_{0}^{2} d z .
\end{aligned}
$$


Dividing (2.25) by $(2.26)$, we obtain

$$
\frac{d y_{0}}{d x}=\frac{\gamma U}{a_{0}}
$$

where we have used $a_{0}^{2}=\gamma p_{0} / \rho_{0}$. Eliminating $d t$ from (2.25) and (2.27), using $a_{0}^{2}=$ $\gamma p_{0} / \rho_{0}$, and integrating the resulting equation subject to the condition $z=0$ at $y=0$, we get

$$
\left.z(x, y)\right|_{y=y_{0}(x)}=\frac{1}{\gamma(\gamma-1)} y_{0}(x) .
$$

Equations (2.9), (2.21), and (2.28) lead to

$$
\left.\frac{\partial z}{\partial x}\right|_{y=y_{0}(x)}=2 \frac{\gamma^{2}-y_{0}^{\prime 2}}{\gamma(\gamma+1) y_{0}^{\prime}} .
$$

Thus, along the shock curve $y=y_{0}(x)$ in the $(x, y)$-plane, the conditions $(2.28)-(2.30)$ must hold.

Now we consider the motion of a piston produced by a gas pressure, with the velocity of the piston equal to the gas velocity $V(t)$ there. Therefore, we have

$$
m \frac{d V}{d t}=-p, \quad V(0)=u_{0}
$$

where $u_{0}$ is the initial velocity of the gas. Integrating (2.31) and using (2.6) at $y=0$, we obtain

$$
z(x, 0)=\frac{1}{2} x^{2}-\frac{u_{0}}{a_{0}} x
$$

The strong shock condition is

$$
z(0, y)=0, \quad y \geq 0
$$

Thus, the boundary value problem for the second-order nonlinear hyperbolic partial differential equation (2.20) has been posed. The function $z(x, y)$ must satisfy the conditions $(2.29)-(2.30)$ at the shock curve $y=y_{0}(x)$, and (2.32) at the piston $y=0$. The third condition would make it possible to determine the shock locus $y=y_{0}(x)$, so that the problem is well-posed.

Since the sound speed $a^{2}=\gamma p / \rho$, Eq. (2.10) gives

$$
a^{2}=\gamma(\gamma-1) a_{0}^{2}\left(z_{y}+\frac{1}{2} z_{x}^{2}\right)
$$

Using the relation $a^{2}=\gamma p / \rho$ in (2.7), the density is found in the form

$$
\rho=\frac{a^{2 /(\gamma-1)}}{\gamma^{1 /(\gamma-1)}(\gamma-1)^{\gamma /(\gamma-1)}}[f(y)]^{-\gamma /(\gamma-1)} .
$$


3. Intermediate integrals. We assume that there exists an intermediate integral of Eq. (2.20) in the form

$$
q=I(x, y, z, p)
$$

with the notation $p=z_{x}$ and $q=z_{y}$. Differentiating (3.1) with respect to $x$ and $y$ to obtain, respectively, $\partial^{2} z / \partial x \partial y$ and $\partial^{2} z / \partial y^{2}$ and using them in (2.20), we get

$$
\begin{aligned}
I_{y}+q I_{z}+\left(I_{x}+p I_{z}\right) & \left(2 p+I_{p}\right)-\phi(y)\left(q+\frac{1}{2} p^{2}\right) \\
+ & {\left[I_{p}^{2}+2 p I_{p}-n\left(q+\frac{1}{2} p^{2}\right)+p^{2}\right] \frac{\partial^{2} z}{\partial x^{2}}=0, \quad n=\frac{\gamma-1}{\gamma} . }
\end{aligned}
$$

Therefore, the necessary conditions for (3.1) to be an intermediate integral of $(2.20)$ are

$$
\begin{aligned}
I_{y}+I I_{z}+\left(I_{x}+p I_{z}\right)\left(2 p+I_{p}\right)-\phi(y)\left(q+\frac{1}{2} p^{2}\right) & =0 \\
I_{p}^{2}+2 p I_{p}-n\left(q+\frac{1}{2} p^{2}\right)+p^{2} & =0 .
\end{aligned}
$$

Solving (3.4) for $I_{p}$ and integrating with respect to $p$, we obtain

$$
q \equiv I=\left[F(x, y, z) \pm \frac{\sqrt{n}}{2} p\right]^{2}-\frac{1}{2} p^{2}
$$

where $F(x, y, z)$ is a function of integration. Replacing $p$ by the first of $(2.9)$ and inserting the definition $c=a_{0} \sqrt{\gamma(\gamma-1)} \sqrt{q+(1 / 2) p^{2}}$, Eq. (3.5) gives

$$
u \pm \frac{2 c}{\gamma-1}=\frac{2 a_{0}}{\sqrt{n}} F(x, y, z) .
$$

For the isentropic case the Riemann invariants are given by (3.6) with $F$ a constant. Therefore (3.6) elegantly generalizes the definition of Riemann invariants to the nonisentropic case. To obtain $F(x, y, z)$, we substitute (3.5) in (3.3) and equate the coefficients of different powers of $p$ to zero; we have

$$
\begin{array}{r}
\frac{\partial F}{\partial y} \pm \sqrt{n} F \frac{\partial F}{\partial x}-\frac{1}{2} \phi(y) F=0 \\
\left(\frac{n}{2}+1\right) \frac{\partial F}{\partial x} \mp \frac{\sqrt{n}}{4} \phi(y)=0
\end{array}
$$

and

$$
F_{z}=0 \text {. }
$$

Integrating the second of (3.7) with respect to $x$, we get

$$
F(x, y)= \pm \frac{n_{0}}{2} x \phi(y)+\frac{1}{2} K(y), \quad n_{0}=\frac{\sqrt{n}}{(n+2)}
$$

where $K(y)$ is a function of integration. On inserting (3.8) into the first of (3.7) and equating the coefficients of different powers of $x$ to zero, we have

$$
\frac{d \phi}{d y}+l_{0} \phi^{2}=0 \quad \text { and } \quad \frac{d K}{d y}+l_{0} \phi K=0, \quad l_{0}=-\frac{1}{n+2} .
$$


A solution of the system (3.9) is

$$
\phi(y)=\left(\alpha_{0}+l_{0} y\right)^{-1} \quad \text { and } \quad K(y)=\beta_{0}\left(\alpha_{0}+l_{0} y\right)^{-1}
$$

where $\alpha_{0}$ and $\beta_{0}$ are arbitrary constants. On inserting (3.8) and (3.10), Eqs. (3.5) and (3.6) give the following intermediate integrals and the corresponding Riemann invariants for the non-isentropic case:

$$
\begin{aligned}
q & =\frac{1}{4}\left[\frac{\beta_{0} \pm n_{0} x}{\alpha_{0}+l_{0} y} \pm \sqrt{n} p\right]^{2}-\frac{1}{2} p^{2} \\
u \pm \frac{2 c}{\gamma-1} & =\frac{a_{0}}{\sqrt{n}} \frac{\beta_{0}+n_{0} x}{\alpha_{0}+l_{0} y} .
\end{aligned}
$$

Now we rewrite the first integral (3.11), with plus sign, in the form

$$
l_{0} \frac{\partial z}{\partial Y}+\alpha_{1}\left(\frac{\partial z}{\partial X}\right)^{2}-\beta_{1} \frac{X}{Y} \frac{\partial z}{\partial X}-\frac{X^{2}}{4 Y^{2}}=0
$$

where $X=\beta_{0}+n_{0} x, Y=\alpha_{0}+l_{0} y, \alpha_{1}=\left[n(2-n) /\left(4(n+2)^{2}\right)\right]$, and $\beta_{1}=[n /(2(n+2))]$. Some solutions of the first-order partial differential equation (3.13) are obtained below.

1. We apply Charpit's method to Eq. (3.13). Writing the characteristic equations for (3.13), we have

$$
\begin{aligned}
& \frac{d X}{d s}=\beta_{1} Y^{-1} X-2 \alpha_{1} p, \quad \frac{d Y}{d s}=-l_{0}, \quad \frac{d z}{d s}=\beta_{1} Y^{-1} X p-2 \alpha_{1} p^{2}-l_{0} q, \\
& \frac{d p}{d s}=-\frac{1}{2} Y^{-2} X-\beta_{1} Y^{-1} p, \quad \frac{d q}{d s}=\frac{1}{2} Y^{-3} X^{2}+\beta_{1} Y^{-2} X_{p},
\end{aligned}
$$

where $s$ is a parameter measured along the characteristic. A solution of the system (3.14) is

$$
\begin{aligned}
X(s) & =\frac{\gamma+1}{3 \gamma-1} c_{0}-\frac{\gamma-1}{3 \gamma-1} c_{1} S \\
Y(s) & =S \\
z(s) & =\frac{(\gamma-1)^{2}}{2 \gamma(3 \gamma-1)} c_{0} c_{1} \log S-\frac{\gamma-1}{3 \gamma-1} c_{1}^{2} S+\frac{\gamma+1}{2(3 \gamma-1)} c_{0}^{2} S^{-1}+c_{4} \\
p(s) & =c_{0} S^{-1}+c_{1} \\
q(s) & =\frac{\gamma-1}{3 \gamma-1} c_{0} c_{1} S^{-1}-\frac{\gamma+1}{2(3 \gamma-1)} c_{0}^{2} S^{-2} \\
S & =\left(\frac{\gamma}{3 \gamma-1} s+c_{3}\right)
\end{aligned}
$$

where $c_{0}, c_{1}, c_{3}$, and $c_{4}$ are arbitrary constants. An initial value problem may be posed for (3.13) and solved with the help of the solution (3.15). Eliminating $s$ from $X, Y$, and $p$, we obtain

$$
\frac{\gamma-1}{2 \gamma} Y \frac{\partial z}{\partial X}+\frac{3 \gamma-1}{2 \gamma} X-c_{1}=0 .
$$


Integrating the relation $d z=p d x+q d y$ with $p$ and $q$ from (3.13) and (3.16), we obtain the following solution of $(2.20)$ :

$$
\begin{aligned}
z(x, y)=\left(\alpha_{0}-\frac{\gamma}{(3 \gamma-1)} y\right)^{-1}[- & \frac{\gamma}{2(3 \gamma-1)} x^{2}+\left(c_{1} \frac{\sqrt{\gamma(\gamma-1)}}{3 \gamma-1}-\beta_{0} \sqrt{\frac{\gamma}{\gamma-1}}\right) x \\
& \left.+c_{1} \beta_{0}-\frac{3 \gamma-1}{2(\gamma-1)} \beta_{0}^{2}-\frac{\gamma^{2}-1}{4 \gamma(3 \gamma-1)} c_{1}^{2}\right]
\end{aligned}
$$

The sound speed is easily obtained by using (3.17) in (2.34):

$$
a^{2}=\gamma(\gamma-1) a_{0}^{2}\left[\frac{c_{1}(\gamma-1)}{4(3 \gamma-1)}\right]^{2}\left(\alpha_{0}-\frac{\gamma}{(3 \gamma-1)} y\right)^{-2} .
$$

The density is obtained from (2.35), (3.10), and (3.17):

$$
\rho=\frac{a^{2 /(\gamma-1)}}{\gamma^{1 /(\gamma-1)}(\gamma-1)^{\gamma /(\gamma-1)}}\left(\alpha_{0}-\frac{\gamma}{(3 \gamma-1)} y\right)^{(3 \gamma-1) /(\gamma-1)} .
$$

2. Another distinct solution of (3.13) may be obtained by writing $z(x, y)=Y^{m_{1}} g(\xi)$, $\xi=X Y^{m_{2}}$. Therefore, it follows from (3.13) that, if $m_{1}=1, m_{2}=-1$, then $g(\xi)$ satisfies the ordinary differential equation

$$
g^{\prime 2}+\frac{2(n+2)}{n} \xi g^{\prime}+\frac{4(n+2)^{2}}{n(n-2)}\left(\frac{1}{n+2} g+\frac{1}{4} \xi^{2}\right)=0
$$

A solution of $(3.20)$ is

$$
g(\xi)=c_{4}^{2} \pm\left[-\frac{4(n+2)}{n(n-2)}\right]^{1 / 2} c_{4} \xi-\frac{n+2}{2(n-2)} \xi^{2},
$$

where $c_{4}$ is a constant. Thus, the solution of $(2.20)$ in the present case is

$$
\begin{aligned}
z(x, y)= & \frac{\gamma(\gamma-1)}{2(\gamma+1)(3 \gamma-1)}\left(\alpha_{0}-\frac{\gamma}{(3 \gamma-1)} y\right)^{-1} x^{2} \\
& +\left[ \pm \frac{2 c_{4} \gamma}{\sqrt{(\gamma+1)(3 \gamma-1)}}+\beta_{0} \frac{\sqrt{\gamma(\gamma-1)}}{\gamma+1}\left(\alpha_{0}-\frac{\gamma}{(3 \gamma-1)} y\right)^{-1}\right] x \\
& +c_{4}^{2}\left(\alpha_{0}-\frac{\gamma}{(3 \gamma-1)} y\right)+\frac{3 \gamma-1}{2(\gamma+1)} \beta_{0}^{2}\left(\alpha_{0}-\frac{\gamma}{(3 \gamma-1)} y\right)^{-1} \\
& \pm c_{4} \beta_{0} \sqrt{\frac{4 \gamma(3 \gamma-1)}{\gamma^{2}-1}}
\end{aligned}
$$

In this case, the density is again given by (3.19) while the sound speed is found to be

$$
\begin{array}{r}
a^{2}=\gamma(\gamma-1) a_{0}^{2}\left[-\frac{\gamma}{\gamma+1}\left(\alpha_{0}-\frac{\gamma}{(3 \gamma-1)} y\right)^{-1}\left(\beta_{0}+\sqrt{\left.\frac{\gamma(\gamma-1)}{3 \gamma-1} x\right)}\right.\right. \\
\left. \pm c_{4} \sqrt{\frac{\gamma^{2}-\gamma}{3 \gamma^{2}+2 \gamma-1}}\right]^{2}
\end{array}
$$


It is interesting to note that the solution (3.22) generalizes the corresponding isentropic solution found by Ustinov [16]:

$$
z(x, y)=\frac{\gamma}{(\gamma+1)^{2}} \frac{[(\gamma+1) x+2]^{2}}{(\gamma+1) y+2 \gamma}-\frac{2}{\gamma+1} x+\frac{2}{\gamma^{2}-1} y-\frac{2}{(\gamma+1)^{2}}
$$

This solution describes a rarefaction wave between a certain moving piston and a leading front.

4. Classical Lie group method. We now seek the following group of infinitesimal transformations (see Bluman and Cole [18]) which takes the $(x, y, z)$ space into itself and under which $(2.20)$ is invariant:

$$
x^{*}=x+\varepsilon X, \quad y^{*}=y+\varepsilon Y, \quad z^{*}=z+\varepsilon Z,
$$

where the generators $X, Y$, and $Z$ are functions of $x, y$, and $z$. Invariance of Eq. (2.20) under (4.1) gives

$$
\begin{aligned}
& Z_{y y}+\left(2 Z_{y z}-Y_{y y}\right) z_{y}-X_{y y} z_{x}+\left(Z_{z z}-2 Y_{y z}\right) z_{y}^{2}-2 X_{y z} z_{x} z_{y}-Y_{z z} z_{y}^{3}-X_{z z} z_{y}^{2} z_{x} \\
& +\left(Z_{z}-2 Y_{z}\right)\left[\phi(y)\left(z_{y}+\frac{1}{2} z_{x}^{2}\right)+\frac{\gamma-1}{\gamma} z_{y} z_{x x}-\frac{\gamma+1}{2 \gamma} z_{x}^{2} z_{x x}-2 z_{x} z_{x y}\right]-2 X_{y} z_{x y} \\
& -3 \phi(y) Y_{z} z_{y}\left(z_{y}+\frac{1}{2} z_{x}^{2}\right)-3 \frac{\gamma-1}{\gamma} Y_{z} z_{y}^{2} z_{x x}+3 \frac{\gamma+1}{2 \gamma} Y_{z} z_{y} z_{x}^{2} z_{x x}+6 Y_{z} z_{x} z_{y} z_{x y} \\
& -\phi(y) X_{z} z_{x}\left(z_{y}+\frac{1}{2} z_{x}^{2}\right)-\frac{\gamma-1}{\gamma} X_{z} z_{x} z_{y} z_{x x}+\frac{\gamma+1}{2 \gamma} X_{z} z_{x}^{3} z_{x x}+2 X_{z} z_{x}^{2} z_{x y}-2 X_{z} z_{y} z_{x y} \\
& +2 z_{x}\left[Z_{x y}+\left(Z_{y z}-X_{x y}\right) z_{x}+\left(Z_{x z}-Y_{x y}\right) z_{y}-X_{y z} z_{x}^{2}+\left(Z_{z z}-Y_{y z}-X_{x z}\right) z_{x} z_{y}\right. \\
& -Y_{x z} z_{y}^{2}-X_{z z} z_{y} z_{x}^{2}-Y_{z z} z_{x} z_{y}^{2}-X_{y} z_{x x}+\left(Z_{z}-X_{x}-Y_{y}\right) z_{x y} \\
& -\phi(y) Y_{x}\left(z_{y}+\frac{1}{2} z_{x}^{2}\right)-\frac{\gamma-1}{\gamma} Y_{x} z_{y} z_{x x}+\frac{\gamma+1}{2 \gamma} Y_{x} z_{x}^{2} z_{x x}+2 Y_{x} z_{x} z_{x y} \\
& -2 X_{z} z_{x} z_{x y}-\phi(y) Y_{z} z_{x}\left(z_{y}+\frac{1}{2} z_{x}^{2}\right)-\frac{\gamma-1}{\gamma} Y_{z} z_{x} z_{y} z_{x x}+\frac{\gamma+1}{2 \gamma} Y_{z} z_{x}^{3} z_{x x} \\
& \left.+2 Y_{z} z_{x}^{2} z_{x y}-X_{z} z_{y} z_{x x}-2 Y_{z} z_{y} z_{x y}\right] \\
& +2 z_{x y}\left[Z_{x}+\left(Z_{z}-X_{x}\right) z_{x}-X_{z} z_{x}^{2}-Y_{x} z_{y}-Y_{z} z_{x} z_{y}\right]+\frac{\gamma+1}{2 \gamma} z_{x}^{2}\left[Z_{x x}+\left(2 Z_{x z}-X_{x x}\right) z_{x}\right. \\
& -Y_{x x} z_{y}+\left(Z_{z z}-2 X_{x z}\right) z_{x}^{2}-2 Y_{x z} z_{x} z_{y}-X_{z z} z_{x}^{3}-Y_{z z} z_{x}^{2} z_{y}+\left(Z_{z}-2 X_{x}\right) z_{x x}-2 Y_{x} z_{x y} \\
& \left.-3 X_{z} z_{x} z_{x x}-Y_{z} z_{y} z_{x x}-2 Y_{z} z_{x} z_{x y}\right] \\
& +\left[\frac{\gamma+1}{\gamma} z_{x} z_{x x}-\phi(y) z_{x}\right]\left[Z_{x}+\left(Z_{z}-X_{x}\right) z_{x}-X_{z} z_{x}^{2}-Y_{x} z_{y}-Y_{z} z_{x} z_{y}\right]
\end{aligned}
$$




$$
\begin{aligned}
& -\frac{\gamma-1}{\gamma} z_{y}\left[Z_{x x}+\left(2 Z_{x z}-X_{x x}\right) z_{x}-Y_{x x} z_{y}+\left(Z_{z z}-2 X_{x z}\right) z_{x}^{2}-2 Y_{x z} z_{x} z_{y}\right. \\
& \left.-X_{z z} z_{x}^{3}-Y_{z z} z_{x}^{2} z_{y}+\left(Z_{z}-2 X_{x}\right) z_{x x}-2 Y_{x} z_{x y}-3 X_{z} z_{x} z_{x x}-Y_{z} z_{y} z_{x x}-2 Y_{z} z_{x} z_{x y}\right] \\
& -\left[\frac{\gamma-1}{\gamma} z_{x x}+\phi(y)\right]\left[Z_{y}+\left(Z_{z}-Y_{y}\right) z_{y}-X_{y} z_{x}-X_{z} z_{x} z_{y}-Y_{z} z_{y}^{2}\right] \\
& -\phi^{\prime}(y)\left[Y_{y z}+\frac{1}{2} Y z_{x}^{2}\right]=0
\end{aligned}
$$

Equating the coefficients of $z_{x}^{4} z_{x x}, z_{x}^{4}$, and $z_{x x}$ in (4.2) to zero, we get

$$
Y_{z}=Z_{z z}=Z_{y}=0 \text {. }
$$

The coefficients of $z_{x}^{3} z_{x x}$ and $z_{y} z_{x y}$ in (4.2) when equated to zero give

$$
2 X_{z}-Y_{x}=0 \quad \text { and } 2 X_{z}+\frac{2}{\gamma} Y_{x}=0
$$

implying that

$$
X_{z}=Y_{x}=0
$$

Again equating the coefficients of $4 z_{x}^{2}, z_{x} z_{x y}, z_{x}^{3}, z_{x y}, z_{x} z_{y}, z_{x}$, and $z_{y}$ in (4.2) to zero and making use of (4.3) and (4.5), we get the determining equations

$$
\begin{aligned}
\frac{1}{2} \phi(y)\left(2 X_{x}-2 Y_{y}-Z_{z}\right)-2 X_{x y}+\frac{\gamma+1}{2 \gamma} Z_{x x}-\frac{1}{2} \phi^{\prime}(y) Y & =0 \\
2 X_{x}-Y_{y}-Z_{z} & =0 \\
2 Z_{x z}-X_{x x} & =0 \\
X_{y}-Z_{x} & =0, \\
2 Z_{x z}+(\gamma-1) X_{x x} & =0, \\
X_{y y}-\phi(y)\left(X_{y}-Z_{x}\right) & =0 \\
Y_{y y}+\phi(y) Y_{y}+\phi^{\prime}(y) Y+\frac{\gamma-1}{\gamma} Z_{x x} & =0 .
\end{aligned}
$$

Using (4.9) in (4.11) we get

$$
X_{y y}=0
$$

Equations (4.8) and (4.10) imply that

$$
Z_{x z}=X_{x x}=0
$$

Equations (4.5), (4.13), and (4.14) lead to

$$
X=k_{1} x y+a_{1} x+a_{2} y+a_{3}
$$

where $k_{1}, a_{1}, a_{2}$, and $a_{3}$ are arbitrary constants. In view of (4.3) and (4.14), (4.9) integrates with respect to $x$ to give

$$
Z=\frac{k_{1}}{2} x^{2}+a_{2} x+a_{4} z+a_{5}
$$


where $a_{4}$ and $a_{5}$ are arbitrary constants. Substituting (4.15) and (4.16) in (4.7) and integrating with respect to $y$ we obtain

$$
Y=k_{1} y^{2}+\left(2 a_{1}-a_{4}\right) y+a_{6},
$$

where $a_{6}$ is a constant. Using (4.7), Eq. (4.6) can be rewritten as

$$
\frac{1}{2} \phi(y) Y_{y}+2 X_{x y}-\frac{\gamma+1}{2 \gamma} Z_{x x}+\frac{1}{2} \phi^{\prime}(y) Y=0 .
$$

Using (4.15)-(4.17) in (4.18) and integrating with respect to $y$ we get

$$
\phi(y)=\frac{a_{7}-k_{1}[(3 \gamma-1) / \gamma] y}{k_{1} y^{2}+\left(2 a_{1}-a_{4}\right) y+a_{6}}
$$

where $a_{7}$ is a constant. It is easily checked that Eq. (4.12) is automatically satisfied when (4.15)-(4.17) and (4.19) are used in it.

On inserting (4.15)-(4.17), the invariant surface condition for $z$, namely, $d x / X=$ $d y / Y=d z / Z$ becomes

$$
\frac{d x}{k_{1} x y+a_{1} x+a_{2} y+a_{3}}=\frac{d y}{k_{1} y^{2}+\left(2 a_{1}-a_{4}\right) y+a_{6}}=\frac{d z}{\left(k_{1} / 2\right) x^{2}+a_{2} x+a_{4} z+a_{5}} .
$$

We now consider a few special cases of (4.20).

1. $k_{1}=a_{4}=a_{2}=a_{5}=0$.

Integration of Eqs. (4.20) gives a similarity solution of (2.20) in the form

$$
z(x, y)=F(\eta), \quad \eta=\frac{a_{1} x+a_{3}}{\sqrt{2 a_{1} y+a_{6}}}
$$

Putting (4.21) in (2.20) we get the following ordinary differential equation for the similarity function $F(\eta)$ :

$$
\eta^{2} F^{\prime \prime}-\frac{\gamma+1}{\gamma} a_{1} \eta F^{\prime} F^{\prime \prime}+\left(\frac{\gamma+1}{2 \gamma}\right) a_{1}^{2} F^{2} F^{\prime \prime}-\left(2 a_{1}+\frac{a_{7}}{2}\right) F^{\prime 2}+\left(3+\frac{a_{7}}{a_{1}}\right) \eta F^{\prime}=0 .
$$

A local balance argument shows that we may seek a solution of (4.22) in the form

$$
F(\eta)=C_{2} \eta^{2}+C_{1} \eta+C_{0}
$$

where the constants $C_{2}, C_{1}$, and $C_{0}$ are to be determined. Substituting (4.23) in (4.22) and equating the coefficients of different powers of $\eta$ to zero, we get

$$
\begin{aligned}
C_{1}^{2}\left[\frac{\gamma+1}{\gamma} a_{1}^{2} C_{2}-2 a_{1}-\frac{a_{7}}{2}\right] & =0, \\
2 \frac{\gamma+1}{\gamma} a_{1}^{2} C_{2}^{2}-\left[2 \frac{\gamma+1}{\gamma} a_{1}+4 a_{1}+a_{7}\right] C_{2}+\frac{a_{7}}{a_{1}}+4 & =0, \\
4 \frac{\gamma+1}{\gamma} a_{1}^{2} C_{2}^{2}-2\left[\frac{\gamma+1}{\gamma} a_{1}+4 a_{1}+a_{7}\right] C_{2}+\frac{a_{7}}{a_{1}}+3 & =0 .
\end{aligned}
$$


Equation (4.25) is satisfied if $C_{2}=1 / a_{1}$. Equation (4.26) then gives $a_{7}=[3-(2 / \gamma)] a_{1}$. Equation (4.24) now yields $C_{1}=0$. The constant $C_{0}$ is arbitrary. Therefore, (4.23) becomes

$$
F(\eta)=\frac{1}{a_{1}} \eta^{2}+C_{0}
$$

Substituting (4.27) in (4.21), we obtain the following solution of $(2.20)$ :

$$
z(x, y)=\frac{1}{a_{1}}\left(\frac{a_{1} x+a_{3}}{\sqrt{2 a_{1} y+a_{6}}}\right)^{2}+C_{0}
$$

To fit a strong shock to the solution (4.28), we assume $a_{3}=0$ in (4.21). The strong shock condition (2.33) and Eq. (4.21) imply $F(0)=0$. It follows from $(4.27)$ and $F(0)=0$ that $C_{0}=0$. From Eq. (4.22) and $F(0)=0$ we have $F^{\prime}(0)=0$. Thus the solution of (2.20) satisfying the strong shock condition (2.33) is

$$
z(x, y)=F(\eta)=\frac{a_{1} x^{2}}{2 a_{1} y+a_{6}}
$$

2. $k_{1}=a_{2}=0$.

In this case, it is easy to verify that the solution of $(2.20)$ has the form

$$
z(x, y)=-\frac{a_{5}}{a_{4}}+\left(a_{1} x+a_{3}\right)^{a_{4} / a_{1}} F(\eta), \quad \eta(x, y)=\frac{a_{1} x+a_{3}}{\left[\left(2 a_{1}-a_{4}\right) y+a_{6}\right]^{a_{1} /\left(2 a_{1}-a_{4}\right)}}
$$

Let $a_{4}=a_{1}, a_{3}=a_{2}=a_{5}=a_{6}=0$. Then

$$
z(x, y)=a_{1} x F(\eta) \text { and } \eta=x / y
$$

Equation (2.20) now transforms to

$$
\begin{gathered}
{\left[a_{1} \eta^{2}-\frac{\gamma+1}{\gamma} a_{1}^{2} \eta^{2} F^{\prime}-2 a_{1}^{2} \eta F+\frac{\gamma+1}{2 \gamma} a_{1}^{3} F^{2}+\frac{\gamma+1}{2 \gamma} a_{1}^{3} \eta^{2} F^{2}+a_{1}^{3} \frac{\gamma+1}{\gamma} \eta F F^{\prime}\right] F^{\prime \prime}} \\
+\frac{\gamma+1}{\gamma} a_{1}^{3} \eta^{-1} F^{2} F^{\prime}-a_{1}\left(2 a_{1} \frac{\gamma+1}{\gamma}+\frac{a_{7}}{2}\right) \eta F^{\prime 2}-a_{1}\left(4 a_{1}+a_{7}\right) F F^{\prime} \\
+2 \frac{\gamma+1}{\gamma} a_{1}^{3} F F^{\prime 2}+\frac{\gamma+1}{\gamma} a_{1}^{3} \eta F^{\prime 3}+\left(2 a_{1}+a_{7}\right) \eta F^{\prime}-\frac{a_{1} a_{7}}{2} \eta^{-1} F^{2}=0
\end{gathered}
$$

The dominant balance argument shows that (4.32) has a solution that is linear in $\eta$. It is easily found to be

$$
F(\eta)=\frac{1}{2 a_{1}} \eta
$$

Thus the solution of $(2.20)$ in this case is

$$
z(x, y)=\frac{x^{2}}{2 y}
$$


Now let $\eta=\eta_{0}$ be a shock of finite strength so that $y=x / \eta_{0}=y_{0}(x)$. Then, Eqs. (2.29)-(2.30) give

$$
F\left(\eta_{0}\right)=\frac{1}{a_{1} \eta_{0} \gamma(\gamma-1)} \quad \text { and } \quad F\left(\eta_{0}\right)+\eta_{0} F^{\prime}\left(\eta_{0}\right)=\frac{2\left(\gamma^{2} \eta_{0}^{2}-1\right)}{a_{1} \eta_{0} \gamma(\gamma+1)}
$$

Solving (4.35) for $F^{\prime}\left(\eta_{0}\right)$, we get

$$
F^{\prime}\left(\eta_{0}\right)=\frac{2 \gamma^{3} \eta_{0}^{2}-2 \gamma^{2} \eta_{0}^{2}-3 \gamma+1}{a_{1} \eta_{0}^{2} \gamma\left(\gamma^{2}-1\right)}
$$

Equations (4.35) and (4.36) give $\eta_{0}=\sqrt{2 /(\gamma(\gamma-1))}$. The shock velocity as obtained from $(2.28)$ is

$$
U=\frac{a_{0}}{\gamma} y_{0}^{\prime}(x)=a_{0} \sqrt{\frac{\gamma-1}{2 \gamma}}=\text { constant. }
$$

3. $k_{1}=a_{2}=a_{4}-2 a_{1}=0$.

The characteristic equations (4.20) now integrate to give

$$
z(x, y)=-\frac{a_{5}}{2 a_{1}}+e^{2 a_{1} y / a_{6}} F(\eta), \quad \eta(x, y)=\left(a_{1} x+a_{3}\right) e^{-a_{1} y / a_{6}}
$$

In this case, (2.20) transforms to

$$
\begin{aligned}
{\left[\eta^{2}+a_{1}^{2} \frac{\gamma+1}{2 \gamma} F^{\prime 2}-a_{1} \frac{\gamma+1}{\gamma} \eta F^{\prime}-2 a_{1}\right.} & \left.\frac{\gamma-1}{\gamma} F\right] F^{\prime \prime}+\left(2 a_{1}-\frac{a_{7}}{2}\right) F^{\prime 2} \\
& +\left(\frac{a_{7}}{a_{1}}-3\right) \eta F^{\prime}+2\left(2-\frac{a_{7}}{a_{1}}\right) F=0 .
\end{aligned}
$$

It is easy to check that

$$
F(\eta)=\frac{1}{2 a_{1}} \eta^{2}+a_{8}
$$

with $a_{8}$ a constant, is a solution of (4.39). Thus the solution of (2.20) as obtained from (4.38) and (4.40) is

$$
z(x, y)=-\frac{a_{5}}{2 a_{1}}+e^{2 a_{1} y}\left(\frac{1}{2 a_{1}}\left(a_{1} x+a_{3}\right)^{2} e^{-2 a_{1} y}+a_{8}\right) .
$$

Now if we take $a_{5}=a_{3}=0$, the strong shock condition (2.33) and (4.38) with $a_{5}=a_{3}=0$ imply that $F(0)=0$. Therefore, it follows from (4.40) and $F(0)=0$ that $a_{8}=0$. Thus, the solution of (2.20) satisfying the strong shock condition $(2.33)$ is $z(x, y)=a_{1} x^{2} / 2$. In this case the sound speed and the density as obtained from (2.34)-(2.35) and (4.41) are

$$
a^{2}=\frac{1}{2} \gamma(\gamma-1)\left(a_{0} a_{1} x\right)^{2} \quad \text { and } \quad \rho=\frac{a^{2 /(\gamma-1)}}{\gamma^{1 /(\gamma-1)}(\gamma-1)^{\gamma /(\gamma-1)}} y^{\left(-\gamma a_{7}\right) /\left(a_{1}(\gamma-1)\right)} \text {. }
$$

4. $k=0, c k_{1}=a b, a^{2}=d k_{1}$, and $b^{2}=2 l k_{1}$.

The characteristic equations (4.20) in this case are easily integrated. We get the solution

$$
z(x, y)=\frac{\left(x+b / k_{1}\right)^{2}}{2\left(y+a / k_{1}\right)}+F(\eta), \quad \eta(x, y)=\frac{x+b / k_{1}}{y+a / k_{1}} .
$$

On substituting (4.43), Eq. (2.20) reduces to $F^{\prime \prime}=0$. Therefore, $F(\eta)=\lambda_{0}+\lambda_{1} \eta$, where $\lambda_{0}$ and $\lambda_{1}$ are arbitrary constants. Thus the solution of $(2.20)$ in the present case is

$$
z(x, y)=\frac{\left(x+b / k_{1}\right)^{2}}{2\left(y+a / k_{1}\right)}+\lambda_{1} \frac{x+b / k_{1}}{y+a / k_{1}}+\lambda_{0} .
$$


5. Solutions by direct similarity method. Following the work of Clarkson and Kruskal [17], we seek solutions of (2.20) in the form

$$
z(x, y)=\alpha(x, y)+\beta(x, y) H(\eta(x, y)), \quad \beta(x, y) \neq 0
$$

We substitute (5.1) in (2.20) and require the resulting equation to be an ordinary differential equation governing the function $H(\eta)$ :

$$
\begin{aligned}
\left(H^{\prime 2}+\Gamma_{1} H H^{\prime}\right. & \left.+\Gamma_{2} H^{\prime}+\Gamma_{3} H^{2}+\Gamma_{4} H+\Gamma_{5}\right) H^{\prime \prime}+\Gamma_{6} H^{\prime 3}+\left(\Gamma_{7} H+\Gamma_{8}\right) H^{\prime 2} \\
& +\left(\Gamma_{9} H^{2}+\Gamma_{10} H+\Gamma_{11}\right) H^{\prime}+\Gamma_{12} H^{3}+\Gamma_{13} H^{2}+\Gamma_{14} H+\Gamma_{15}=0 .
\end{aligned}
$$

The functions $\Gamma_{n}=\Gamma_{n}(\eta), n=1,2, \ldots, 15$ are introduced according to

$$
\begin{aligned}
2 \beta_{x} & =\beta \Gamma_{1}, \\
2 \omega \alpha_{x} \eta_{x}+(2-n) \eta_{y} & =\omega \beta \eta_{x}^{2} \Gamma_{2}, \\
\beta_{x}^{2} & =\beta^{2} \eta_{x}^{2} \Gamma_{3} \\
2 \omega \alpha_{x} \beta_{x} \eta_{x}+2 \beta_{x} \eta_{y}-n \beta_{y} \eta_{x} & =\omega b t^{2} \eta_{x}^{3} \Gamma_{4} \\
\omega \alpha_{x}^{2} \eta_{x}^{2}+2 \alpha_{x} \eta_{x} \eta_{y}-n \alpha_{y} \eta_{x}^{2}+\eta_{y}^{2} & =\omega \beta^{2} \eta_{x}^{4} \Gamma_{5} \\
\beta \eta_{x x}+2 \beta_{x} \eta_{x} & =\beta \eta_{x}^{2} \Gamma_{6} \\
2 \beta \beta_{x} \eta_{x x}+4 \beta_{x}^{2} \eta_{x}+\beta \beta_{x x} \eta_{x} & =\beta^{2} \eta_{x}^{3} \Gamma_{7} \\
4 \omega \beta \alpha_{x} \eta_{x} \eta_{x x}-2 n \beta \eta_{y} \eta_{x x}+4 \beta \eta_{x} \eta_{x y}+8 \omega \alpha_{x} \beta_{x} \eta_{x} & \\
-4(n-1) \beta_{x} \eta_{x} \eta_{y}+4 \beta_{y} \eta_{x}^{2}+2 \omega \beta \alpha_{x x} \eta_{x}^{2}-\phi(y) \beta \eta_{x}^{2} & =2 \omega \beta^{2} \eta_{x}^{4} \Gamma_{8} \\
\beta_{x}\left(\beta \beta_{x} \eta_{x x}+2 \beta_{x}^{2} \eta_{x}+2 \beta \beta_{x x} \eta_{x}\right) & =\beta^{3} \eta_{x}^{3} \Gamma_{9}
\end{aligned}
$$

$$
\begin{aligned}
& 4 \omega \beta \alpha_{x} \beta_{x} \eta_{x x}-2 n \beta \beta_{y} \eta_{x x}+4 \beta \beta_{x} \eta_{x y}+8 \omega \alpha_{x} \beta_{x} \eta_{x} \\
& \quad+4 \omega \beta \alpha_{x} \beta_{x x} \eta_{x}+4 \beta_{x} \eta_{y}-4(n-1) \beta_{x} \beta_{y} \eta_{x}+4 \omega \beta \alpha_{x x} \beta_{x} \eta_{x} \\
& \quad-2 \phi(y) \beta \beta_{x} \eta_{x}+4 \beta \beta_{x y} \eta_{x}-2 n \beta \beta_{x x} \eta_{y}=2 \omega \beta^{3} \eta_{x}^{4} \Gamma_{10}
\end{aligned}
$$

$$
\begin{aligned}
2 \omega \beta \alpha_{x}^{2} \eta_{x x}+2 n \beta \alpha_{y} \eta_{x x}+2 \beta \eta_{y y}+4 \beta \alpha_{x} \eta_{x y}+4 \omega \alpha_{x}^{2} \beta_{x} \eta_{x} & \\
+4 \alpha_{x} \beta_{x} \eta_{y}+4 \alpha_{x} \beta_{y} \eta_{x}+4 \omega \beta \alpha_{x} \alpha_{x x} \eta_{x}-4 n \alpha_{y} \beta_{x} \eta_{x} & \\
+4 \beta_{y} \eta_{y}+4 \beta \alpha_{x y} \eta_{x}-2 n \beta \alpha_{x x} \eta_{y}-2 \phi(y) \beta\left(\alpha_{x} \eta_{x}+\eta_{y}\right) & =2 \omega \beta^{3} \eta_{x}^{4} \Gamma_{11} \\
\beta_{x}^{2} \beta_{x x} & =\beta^{3} \eta_{x}^{4} \Gamma_{12} \\
4 \omega \alpha_{x} \beta_{x} \beta_{x x}+2 \omega \alpha_{x x} \beta_{x}^{2}+4 \beta_{x} \beta_{x y}-2 n \beta_{y} \beta_{x x}-\phi(y) \beta_{x}^{2} & =2 \omega \beta^{3} \eta_{x}^{4} \Gamma_{13} \\
2 \omega \alpha_{x} \beta_{x x}+4 \omega \alpha_{x} \alpha_{x x} \beta_{x}+4 \alpha_{x} \beta_{x y}-2 n \alpha_{y} \beta_{x x}+4 \beta_{x} \alpha_{x y} & \\
-2 n \alpha_{x x} \beta_{y}+2 \beta_{y y}-2 \phi(y)\left(\alpha_{x} \beta_{x}+\beta_{y}\right) & =2 \omega \beta^{3} \eta_{x}^{4} \Gamma_{14} \\
2 \alpha_{y y}+4 \alpha_{x} \alpha_{x y}+2 \omega \alpha_{x}^{2} \alpha_{x x}-2 n \alpha_{y} \alpha_{x x}-\phi(y)\left(\alpha_{x}^{2}+2 \alpha_{y}\right) & =2 \omega \beta^{3} \eta_{x}^{4} \Gamma_{15}
\end{aligned}
$$

where $\omega=(\gamma+1) /(2 \gamma)=1-n / 2$. 
Now with the help of the following three remarks we solve Eqs. (5.3)-(5.17) for the functions $\alpha(x, y), \beta(x, y), \eta(x, y)$, and $\Gamma_{n}(\eta), n=1,2, \ldots, 15$. Remarks: (1) If $\alpha(x, y)$ has the form $\alpha(x, y)=\hat{\alpha}(x, y)+\beta(x, y) \Omega(\eta)$, then we may choose $\Omega \equiv 0$. (2) If $\beta(x, y)$ is found to have the form $\beta(x, y)=\hat{\beta}(x, y) \Omega(\eta)$, then we may put $\Omega(\eta)=1$. (3) If $\eta(x, y)$ is determined from an equation of the form $f(\eta)=\hat{\eta}(x, y)$, where $f(\eta)$ is any invertible function then we may take $f(\eta)=\eta$ without loss of generality.

Setting $\Gamma_{1}(\eta)=2 \Omega_{1}^{\prime}(\eta) / \Omega_{1}(\eta)$ in (5.3) and integrating with respect to $x$ leads to $\beta=\hat{\beta}(y) \Omega_{1}(\eta)$, where $\hat{\beta}(x, t)>0$ is a function of integration. Using Remark 2 we choose $\Omega_{1}(\eta)=1$ so that

$$
\beta=\beta(y),
$$

where we have dropped the hat on $\beta$. Putting $\Gamma_{6}(\eta)=-\Omega_{6}^{\prime \prime}(\eta) / \Omega_{6}^{\prime}(\eta)$ in (5.8) and integrating twice with respect to $x$ yields $\Omega_{6}(\eta)=x \theta(y)+\psi(y)$, where $\theta(y)$ and $\psi(y)$ are two functions of integration. In view of Remark 3 we choose $\Omega_{6}(\eta)=\eta$ so that $\eta(x, y)=x \theta(y)+\psi(y)$. Hence we obtain

$$
\eta(x, y)=x \theta(y)+\psi(y)
$$

Putting $\Gamma_{2}(\eta)=2 \Omega_{2}^{\prime}(\eta)$ in (5.4) and integrating with respect to $x$ yields $\alpha=\beta \Omega_{2}-$ $(x /(2 \theta))\left(x \theta^{\prime}+2 \psi^{\prime}\right)+\lambda(y)$, where $\lambda(y)$ is a function of integration. Using Remark 1 we choose $\Omega_{2}(\eta)=0$. Thus we have

$$
\alpha(x, y)=-\frac{x}{2 \theta(y)}\left[x \theta^{\prime}(y)+2 \psi^{\prime}(y)\right]+\lambda(y)
$$

It is now easily seen from $(5.3)-(5.5),(5.8),(5.9),(5.11),(5.12),(5.14),(5.15)$, and $(5.18)$ that

$$
\Gamma_{n}(\eta)=0, \quad n=1,2,3,6,7,9,10,12,13 .
$$

Equation (5.6) requires that $\Gamma_{4}(\eta)=k=$ constant so that

$$
\beta^{\prime}=-\omega_{0} k \theta^{2} \beta^{2}, \quad \omega_{0}=\omega / n .
$$

On inserting (5.19)-(5.20), (5.7) becomes

$$
\left(x \theta^{\prime}+\psi^{\prime}\right)^{2}-\theta\left(x^{2} \theta^{\prime \prime}+2 x \psi^{\prime \prime}\right)+\theta^{\prime}\left(x^{2} \theta^{\prime}+2 x \psi^{\prime}\right)+2 \theta^{2} \lambda^{\prime}=-2 \omega_{0} \beta^{2} \theta^{4} \Gamma_{5}(\eta)
$$

Since the left-hand side of (5.23) is quadratic in $x$, the function $\Gamma_{5}(\eta)$ must assume the form $\Gamma_{5}(\eta)=a \eta^{2}+b \eta+c$, where $a, b$, and $c$ are constants; with this form of $\Gamma_{5}(\eta)$, Eq. $(5.23)$ is satisfied provided

$$
\begin{aligned}
\theta \theta^{\prime \prime}-2 \theta^{2} & =2 a \omega_{0} \beta^{2} \theta^{6}, \\
\psi^{\prime \prime}-2 \theta^{-1} \theta^{\prime} \psi^{\prime} & =\omega_{0} \beta^{2} \theta^{4}(2 a \psi+b), \\
2 \theta^{2} \lambda^{\prime}+\psi^{\prime 2} & =-2 \omega_{0} \beta^{2} \theta^{4}\left(a \psi^{2}+b \psi+c\right) .
\end{aligned}
$$

Equation (5.10) requires that $\Gamma_{8}(\eta)=l$ so that

$$
\phi(y)=2(2-\omega) \theta^{-1} \theta^{\prime}-2 \omega\left(2 k_{0}+l\right) \theta^{2} \beta, k_{0}=k / n .
$$


It is easy to verify that, in view of (5.24)-(5.25), Eqs. (5.13) and (5.17) are satisfied provided that $\Gamma_{11}(\eta)=(-1 / n)(2 a \eta+b), \Gamma_{15}(\eta)=-2 \omega_{0}(2 k / n+l)\left(a \eta^{2}+b \eta\right)+d$, and

$$
\lambda^{\prime \prime}-2 \theta^{-1} \theta^{\prime} \lambda^{\prime}=\left[2 k \omega_{0}^{2}\left(a \psi^{2}+b \psi\right)+2 \omega \omega_{0}\left(2 k_{0}+l\right) c+\omega d\right] \theta^{4} \beta^{3}-2 \omega_{0} \beta^{2} \theta^{2} \psi^{\prime}(2 a \psi+b),
$$

where $d$ is a constant. Equation (5.16) requires that $\Gamma_{14}(\eta)=h=$ constant, implying that

$$
\beta^{\prime \prime}-2 \theta^{-1} \theta^{\prime} \beta^{\prime}=\left[h \omega+\left(2 \omega \omega_{0} k\right)(2 k / n+l)\right] \theta^{4} \beta^{3} .
$$

Thus we have derived six equations, namely (5.22), (5.24)-(5.26), (5.28), and (5.29), governing the four functions $\theta(y), \beta(y), \psi(y)$, and $\lambda(y)$. If the constants $h$ and $d$ are chosen to be $h=\left(-2 k \omega_{0}\right)(k / n+l)$ and $d=\left(-2 \omega_{0}\right)(k / n+l) c$, then this overdetermined system becomes determinant in the following sense: Equation (5.29), with $h=\left(-2 k \omega_{0}\right)(k / n+l)$, may be obtained from (5.22) by once differentiating the latter with respect to $y$; similarly, Eq. (5.28), with $d=\left(-2 \omega_{0}\right)(k / n+l) c$, may be obtained from (5.26) by once differentiating the latter with respect to $y$ and using (5.22) and (5.25).

Thus, the solution (5.1) of (2.20) has the form

$$
\begin{aligned}
& z(x, y)=\lambda(y)-\frac{x}{2 \theta(y)}\left[x \theta^{\prime}(y)+2 \psi^{\prime}(y)\right]+\beta(y) H(\eta) \\
& \eta(x, y)=x \theta(y)+\psi(y)
\end{aligned}
$$

where the functions $\beta(y), \theta(y), \psi(y)$, and $\lambda(y)$ are governed by the determinate system of ordinary differential equations (5.22), (5.24)-(5.26).

Substituting for $\Gamma_{n}(\eta), n=1,2, \ldots, 15$, thus found, in (5.2), we obtain the following second-order nonlinear, non-autonomous ordinary differential equation for $H(\eta)$ :

$$
\begin{aligned}
\left(H^{\prime 2}+k H+a \eta^{2}+b \eta+c\right) H^{\prime \prime} & +l H^{\prime 2}-\frac{1}{n}(2 a \eta+b) H^{\prime} \\
& -2 k \omega_{0}\left(k_{0}+l\right) H-2 \omega_{0}\left(k_{0}+l\right)\left(a \eta^{2}+b \eta+c\right)=0 .
\end{aligned}
$$

We may seek a solution of (5.32) in the quadratic form

$$
H(\eta)=A \eta^{2}+B \eta+C
$$

The constants $A, B$, and $C$ are given by the algebraic equations

$$
\begin{array}{r}
4 A^{3}+(2 l+k) A^{2}+\omega\left(a-k_{0}^{2}-l k_{0}\right) A-a \omega_{0}\left(k_{0}+l\right)=0, \\
{\left[4 A^{2}+(2 l+k) A-a / n-k k_{0} \omega_{0}-k l \omega_{0}\right] B+b(1-1 / n) A-b \omega_{0}\left(k_{0}+l\right)=0} \\
2 k\left(A-k_{0} \omega_{0}-l \omega_{0}\right) C+(2 A+l) B^{2}-(b / n) B+2 c A-2 \omega_{0}\left(k_{0}+l\right) c=0 .
\end{array}
$$

A solution of the algebraic equations (5.34)-(5.36) will be obtained for certain simple cases.

Now we return to the system (5.22), (5.24)-(5.26). Equations (5.22) and (5.24) are coupled and nonlinear and once these are solved for the functions $\beta(y)$ and $\theta(y)$, then (5.25) for $\psi(y)$, being linear and inhomogeneous, may be solved. The function $\lambda(y)$ is 
then easily obtained from (5.26). Some simple solutions of this system are obtained below and the solution of $(2.20)$ is written out in each case:

1. A solution of Eqs. (5.22), (5.24)-(5.26) is easily found to be

$$
\begin{aligned}
& \theta(y)=m_{0}\left(m_{1} y+m_{2}\right)^{n_{1}}, \quad \beta(y)=\frac{n m_{1}\left(2 n_{1}+1\right)}{k \omega m_{0}^{2}}\left(m_{1} y+m_{2}\right)^{-2 n_{1}-1} \\
& \psi(y)=-b / 2 a, \quad \lambda(y)=-\frac{\left(b^{2}-4 a c\right) n m_{1}\left(2 n_{1}+1\right)}{4 a \omega k^{2} m_{0}^{2}}\left(m_{1} y+m_{2}\right)^{-2 n_{1}-1}
\end{aligned}
$$

where $m_{0}, m_{1}$, and $m_{2}$ are arbitrary constants and $n_{1}$ satisfies the quadratic $n_{1}^{2}+n_{1}+$ $\left(2 a n /\left(8 a n+\omega k^{2}\right)\right)=0$. On inserting (5.37), Eqs. (5.30)-(5.31) give the following similarity form of the solution of $(2.20)$ :

$$
\begin{aligned}
z(x, y)= & -\frac{\left(b^{2}-4 a c\right) n m_{1}\left(2 n_{1}+1\right)}{4 a \omega k^{2} m_{0}^{2}}\left(m_{1} y+m_{2}\right)^{-2 n_{1}-1}-\frac{1}{2} n_{1} m_{1}\left(m_{1} y+m_{2}\right)^{-1} x^{2} \\
& +\frac{n m_{1}\left(2 n_{1}+1\right)}{k \omega m_{0}^{2}}\left(m_{1} y+m_{2}\right)^{-2 n_{1}-1} H(\eta), \\
\eta(x, y)= & m_{0}\left(m_{1} y+m_{2}\right)^{\eta_{1}} x-b /(2 a) .
\end{aligned}
$$

Equations (5.33) and (5.38) now yield the solution

$$
\begin{aligned}
z(x, y)= & m_{1}\left[\frac{-n_{1}}{2}+\frac{n}{k \omega}\left(1+2 n_{1}\right) m_{0}^{2} A\right]\left(m_{1} y+m_{2}\right)^{-1} x^{2} \\
& +\frac{n}{k \omega}\left(1+2 n_{1}\right) m_{0}^{2}\left[-\frac{b}{a} A+B\right]\left(m_{1} y+m_{2}\right)^{-n_{1}-1} x \\
& +\left[\frac{b^{2} A}{4 a^{2}}-\frac{b B}{a}+C-\frac{b^{2}-4 a c}{4 a k}\right] \frac{n}{k \omega}\left(1+2 n_{1}\right) m_{0}^{2} m_{1}\left(m_{1} y+m_{2}\right)^{-2 n_{1}-1}
\end{aligned}
$$

The function $\phi(y)$ is given by

$$
\phi(y)=2 m_{1}\left[n_{1}(2-\omega)-k_{0}^{-1}\left(2 k_{0}+l\right)\left(1+2 n_{1}\right)\right]\left(m_{1} y+m_{2}\right)^{-1} .
$$

2. $b=c=0$.

In this case, the solution of the system $(5.22),(5.24)-(5.26)$ is given by

$$
\begin{aligned}
& \theta(y)=m_{0} e^{-m_{1} y}, \quad \beta(y)=-\frac{2 n m_{1}}{\omega k m_{0}^{2}} e^{2 m_{1} y}, \quad \psi(y)=d_{0} e^{-m_{1} y} \\
& \lambda(y)=\lambda_{0}-\frac{d_{0}^{2} m_{1}^{2}}{2 m_{0}^{2}}\left(1+\frac{8 a n}{\omega k^{2}}\right) y
\end{aligned}
$$

where $m_{0}, m_{1}, d_{0}$, and $\lambda_{0}$ are arbitrary constants. Using (5.41) in (5.30)-(5.31), we obtain the following similarity form of the solution of $(2.20)$ :

$$
\begin{aligned}
& z(x, y)=\lambda_{0}-\frac{d_{0}^{2} m_{1}^{2}}{2 m_{0}^{2}}\left(1+\frac{8 a n}{\omega k^{2}}\right) y+\frac{1}{2} m_{1}^{2} x^{2}+\frac{m_{1} d_{0}}{m_{0}} x-\frac{2 n m_{1}}{\omega k m_{0}^{2}} e^{2 m_{1} y} H(\eta), \\
& \eta(x, y)=\left(m_{0} x+d_{0}\right) e^{-m_{1} y}
\end{aligned}
$$


Thus, the solution of (2.20) as obtained from (5.33) and (5.42) is

$$
\begin{array}{r}
z(x, y)=\lambda_{0}-\frac{2 n m_{1} d_{0}^{2} A}{\omega k m_{0}^{2}}-\frac{d_{0}^{2} m_{1}^{2}}{2 m_{0}^{2}}\left(1+\frac{8 a n}{\omega k^{2}}\right) y-\frac{2 n m_{1} d_{0}}{\omega k m_{0}^{2}}\left(B+C e^{m_{1} y}\right) e^{m_{1} y} \\
+\frac{m_{1}}{m_{0}}\left[d_{0}-\frac{2 n}{\omega k}\left(2 d_{0} A+B e^{m_{1} y}\right)\right] x+m_{1}\left(\frac{1}{2}-\frac{2 n A}{\omega k}\right) x^{2} .
\end{array}
$$

Here, the function $\phi(y)=2 m_{1}\left(\omega+l k_{0}^{-1}\right)$.

With $a_{1}=m_{0}, m_{1}=a_{1} / a_{6}, \lambda_{0}=-a_{5} /\left(2 a_{1}\right), 8 a n+\omega k^{2}=0$, and $a_{7}=2(\omega+2+$ $\left.2 l k_{0}^{-1}\right) a_{1}$, we recover the similarity form (4.38), obtained by the group-theoretic method provided that

$$
\frac{2 n m_{1}}{\omega k} H(\eta)=-m_{0}^{2} F(\eta)+\frac{m_{1}}{2} \eta^{2}
$$

3. $k=0$ and $b^{2}=4 a c$.

It is easy to check that the solution of Eqs. (5.22), (5.24)-(5.26) is now given by

$$
\begin{aligned}
& \theta(y)=m_{0}\left(m_{1} y+m_{2}\right)^{-1 / 2}, \quad \beta(y)=\frac{m_{1}}{m_{0}^{2}} \sqrt{\frac{n}{8 a \omega}} \\
& \psi(y)=-b /(2 a), \quad \lambda(y)=\lambda_{0},
\end{aligned}
$$

where $m_{0}, m_{1}, m_{2}$, and $\lambda_{0}$ are arbitrary constants. Putting (5.45) in (5.30)-(5.31), we get

$$
\begin{aligned}
& z(x, y)=\lambda_{0}+\frac{1}{4} m_{1}\left(m_{1} y+m_{2}\right)^{-1} x^{2}+\frac{m_{1}}{m_{0}^{2}} \sqrt{\frac{n}{8 a \omega}} H(\eta), \\
& \eta(x, y)=m_{0}\left(m_{1} y+m_{2}\right)^{-1 / 2} x-b /(2 a) .
\end{aligned}
$$

On inserting (5.33), Eq. (5.46) gives the solution

$$
\begin{aligned}
z(x, y)= & m_{1}\left[\frac{1}{4}+A \sqrt{\frac{n}{8 a \omega}}\right]\left(m_{1} y+m_{2}\right)^{-1} x^{2} \\
& +m_{0} m_{1}\left[-\frac{b A}{a}+B\right] \sqrt{\frac{n}{8 a \omega}}\left(m_{1} y+m_{2}\right)^{-1 / 2} x \\
& +\lambda_{0}+m_{0}^{2} m_{1}\left[\frac{b^{2} A}{4 a^{2}}-\frac{b B}{a}+C\right] \sqrt{\frac{n}{8 a \omega}}
\end{aligned}
$$

In this case, the function $\phi(y)$ is given by

$$
\phi(y)=-m_{1}\left[2-\omega+\left(2 k_{0}+l\right) \sqrt{\frac{n \omega}{2 a}}\right]\left(m_{1} y+m_{2}\right)^{-1} .
$$

4. $k=b=c=0$.

Here, the solution of the system $(5.22),(5.24)-(5.26)$ is found to be

$$
\begin{array}{ll}
\theta(y)=m_{0}\left(m_{1} y+m_{2}\right)^{-1 / 2}, & \beta(y)=\frac{m_{1}}{m_{0}^{2}} \sqrt{\frac{n}{8 a \omega}} \\
\psi(y)=d_{0}\left(m_{1} y+m_{2}\right)^{-1 / 2}, & \lambda(y)=\frac{m_{1} d_{0}^{2}}{4 m_{0}^{2}}\left(m_{1} y+m_{2}\right)^{-1},
\end{array}
$$


where $m_{0}, m_{1}, m_{2}$, and $d_{0}$ are arbitrary constants. On using (5.49), Eqs. (5.30)-(5.31) yield

$$
\begin{gathered}
z(x, y)=\frac{1}{2 m_{0}} \eta^{2}+\frac{2}{m_{0}} \sqrt{\frac{n}{8 a \omega}} H(\eta) \\
\eta=\left(m_{0} x+d_{0}\right)\left(m_{1} y+m_{2}\right)^{-1 / 2}
\end{gathered}
$$

Equations (5.33) and (5.50) now lead to the solution

$$
\begin{aligned}
z(x, y)= & m_{1}\left[\frac{1}{4}-\frac{l}{2} \sqrt{\frac{n}{8 a \omega}}\right]\left(m_{1} y+m_{2}\right)^{-1} x^{2} \\
& +\left(d_{0} m_{0} m_{1}\left[\frac{1}{4}-l \sqrt{\frac{n}{8 a \omega}}\right]\left(m_{1} y+m_{2}\right)^{-1}\right) x \\
& +d_{0}\left[\frac{1}{4} m_{0}^{2} m_{1}-\frac{l d_{0}}{2}\right]\left(m_{1} y+m_{2}\right)^{-1}+C,
\end{aligned}
$$

where $C$ is a free constant. The function $\phi(y)$ appearing in $(2.20)$ is now found to be

$$
\phi(y)=-m_{1}\left[2-\omega+\left(2 k_{0}+l\right) \sqrt{\frac{n \omega}{2 a}}\right]\left(m_{1} y+m_{2}\right)^{-1} .
$$

With $m_{1}=2 m_{0}, d_{0}=a_{3}, m_{2}=a_{6}$, and $a_{7}=-2 a_{1}[2+l \omega \sqrt{(-\omega+n /(8 a \omega))}]$, we recover the similarity form $(4.21)$, obtained by the group-theoretic method provided that

$$
F(\eta)=\frac{1}{2 m_{0}} \eta^{2}+\frac{2}{m_{0}} \sqrt{\frac{n}{8 a \omega}} H(\eta)
$$

5. $a=b=c=0$.

In this case, the solution of Eqs. (5.22), (5.24)-(5.26) is given by

$$
\theta(y)=m_{0}\left(m_{1} y+m_{2}\right)^{-1}, \quad \beta(y)=-\frac{n m_{1}}{\omega k m_{0}^{2}}\left(m_{1} y+m_{2}\right), \quad \psi(y)=\lambda(y)=0
$$

where $m_{0}, m_{1}$, and $m_{2}$ are free constants. On inserting (5.54), Eqs. (5.30)-(5.31) give

$$
\begin{aligned}
& z(x, y)=\frac{m_{1}}{2}\left(m_{1} y+m_{2}\right)^{-1} x^{2}-\frac{n m_{1}}{\omega k m_{0}^{2}}\left(m_{1} y+m_{2}\right) H(\eta) \\
& \eta(x, y)=m_{0} x\left(m_{1} y+m_{2}\right)^{-1}
\end{aligned}
$$

Thus, the solution of $(2.20)$ is now given by

$$
z(x, y)=m_{1}\left(\frac{1}{2}-\frac{n A}{\omega k}\right)\left(m_{1} y+m_{2}\right)^{-1} x^{2}-\frac{n m_{1} B}{\omega k m_{0}} x-\frac{n m_{1} C}{\omega k m_{0}^{2}}\left(m_{1} y+m_{2}\right)
$$


where $A$ satisfies the quadratic $4 A^{2}+(2 l+k) A-k \omega_{0}(k / n+l)=0$ and $C=-(1 /(2 k))[(2 l+$ $\left.A) /\left\{A-\omega_{0}(k / n+l)\right\}\right] B^{2}$; the constant $B$ is arbitrary. The function $\phi(y)=2 m_{1}[\omega+$ $\left.k_{0}^{-1} l\right]\left(m_{1} y+m_{2}\right)^{-1}$.

With $m_{0}=m_{1}=1, m_{2}=0$, and $a_{7}=2 k_{0}^{-1}\left(\omega k_{0}+l\right) a_{1}$, we get the similarity form (4.31), obtained by the group-theoretic method, provided that

$$
a_{1} F(\eta)=\frac{1}{2} \eta-\frac{n}{\omega k} \frac{1}{\eta} H(\eta)
$$

6. $a=0$.

In the present case, we obtain two distinct solutions of the system (5.22), (5.24)-(5.26):

(i) One solution of the system $(5.22),(5.24)-(5.26)$ is given by

$$
\begin{gathered}
\theta(y)=m_{0}, \quad \beta(y)=\frac{n m_{1}}{\omega k m_{0}^{2}}\left(m_{1} y+m_{2}\right)^{-1} \\
\psi(y)=d_{1}+d_{0} y-\frac{n b}{\omega k^{2}} \log \left(m_{1} y+m_{2}\right) \\
\lambda(y)=\lambda_{0}-\frac{d_{0}^{2}}{2 m_{0}^{2}} y+\frac{n m_{1}}{\omega k^{2} m_{0}^{2}}\left(c-\frac{n b^{2}}{2 \omega k^{2}}\right)\left(m_{1} y+m_{2}\right)^{-1} \\
+\frac{n b m_{1}}{\omega k m_{0}^{2}}\left(d_{0} y+d_{1}\right)\left(m_{1} y+m_{2}\right)^{-1} \\
-\frac{m_{1} n^{2} b^{2}}{\omega^{2} k^{4} m_{0}^{2}}\left(m_{1} y+m_{2}\right)^{-1} \log \left(m_{1} y+m_{2}\right)
\end{gathered}
$$

where $m_{0}, m_{1}, m_{2}, d_{0}, d_{1}$, and $\lambda_{0}$ are arbitrary constants. Therefore the similarity form of the solution of (2.20) as obtained from (5.30)-(5.31) and (5.58) is

$$
\begin{aligned}
z(x, y)= & \lambda_{0}-\frac{d_{0}^{2}}{2 m_{0}^{2}} y+\frac{n m_{1}}{\omega k^{2} m_{0}^{2}}\left(c-\frac{n b^{2}}{2 \omega k^{2}}\right)\left(m_{1} y+m_{2}\right)^{-1} \\
& +\frac{n b m_{1}}{\omega k m_{0}^{2}}\left(d_{0} y+d_{1}\right)\left(m_{1} y+m_{2}\right)^{-1} \\
& -\frac{m_{1} n^{2} b^{2}}{\omega^{2} k^{4} m_{0}^{2}}\left(m_{1} y+m_{2}\right)^{-1} \log \left(m_{1} y+m_{2}\right) \\
& -\frac{1}{m_{0}}\left(d_{0}-\frac{n b m_{1}}{\omega k^{2}}\left(m_{1} y+m_{2}\right)^{-1}\right) x+\frac{n m_{1}}{\omega k m_{0}^{2}}\left(m_{1} y+m_{2}\right)^{-1} H(\eta), \\
\eta(x, y)= & m_{0} x+d_{1}+d_{0} y-\frac{n b}{\omega k^{2}} \log \left(m_{1} y+m_{2}\right) .
\end{aligned}
$$


Putting (5.33) in (5.59), we obtain

$$
\begin{aligned}
z(x, y)= & \frac{n m_{1} A}{\omega k}\left(m_{1} y+m_{2}\right)^{-1} x^{2} \\
& +\left[\frac{n m_{1}}{\omega k m_{0}}\left(\frac{b}{k}+B-\frac{2 b n A}{\omega k^{2}} \log \left(m_{1} y+m_{2}\right)+2 A\left(d_{0} y+d_{1}\right)\right)\right. \\
& \left.\quad-\frac{d_{0}}{m_{0}}\left(m_{1} y+m_{2}\right)\right]\left(m_{1} y+m_{2}\right)^{-1} x \\
+ & \quad-\frac{n m_{1}}{\omega k m_{0}^{2}}\left[\frac{1}{\omega}\left(c-\frac{n b^{2}}{2 \omega k^{2}}\right)+\frac{b}{k}\left(d_{0} y+d_{1}\right)\right. \\
& \quad+A\left(d_{0} y+d_{1}\right)^{2}-\frac{2 n b A}{\omega k^{2}}\left(d_{0} y+d_{1}\right) \log \left(m_{1} y+m_{2}\right) \\
& \left.\quad-\frac{n b B}{\omega k^{3}} \log \left(m_{1} y+m_{2}\right)+B\left(d_{0} y+d_{1}\right)+C\right]\left(m_{1} y+m_{2}\right)^{-1} \\
& -\frac{d_{0}^{2}}{2 m_{0}^{2}} y+\lambda_{0} .
\end{aligned}
$$

The function $\phi(y)=-\left(2 n m_{1} / k\right)\left(2 k_{0}+l\right)\left(m_{1} y+m_{2}\right)^{-1}$.

(ii) Another solution of the system (5.22), (5.24)-(5.26) is found to be

$$
\begin{aligned}
\theta(y)= & m_{0}\left(m_{1} y+m_{2}\right)^{-1}, \quad \beta(y)=-\frac{n m_{1}}{\omega k m_{0}^{2}}\left(m_{1} y+m_{2}\right) \\
\psi(y)= & d_{1}-\frac{d_{0}}{m_{1}}\left(m_{1} y+m_{2}\right)^{-1}+\frac{n b}{\omega k^{2}} \log \left(m_{1} y+m_{2}\right) \\
\lambda(y)= & \lambda_{0}+\frac{d_{0}^{2}}{2 m_{1} m_{0}^{2}}\left(m_{1} y+m_{2}\right)^{-1}+\frac{n m_{1}}{\omega k^{2} m_{0}^{2}}\left(\frac{n b^{2}}{2 \omega k^{2}}-b d_{1}-c\right)\left(m_{1} y+m_{2}\right) \\
& -\frac{m_{1} n^{2} b^{2}}{\omega^{2} k^{4} m_{0}^{2}}\left(m_{1} y+m_{2}\right) \log \left(m_{1} y+m_{2}\right)
\end{aligned}
$$

where $m_{0}, m_{1}, m_{2}, d_{0}, d_{1}$, and $\lambda_{0}$ are arbitrary constants. On inserting (5.61), Eqs. (5.30)-(5.31) give

$$
\begin{aligned}
z(x, y)= & \lambda_{0}+\frac{d_{0}^{2}}{2 m_{1} m_{0}^{2}}\left(m_{1} y+m_{2}\right)^{-1}+\frac{n m_{1}}{\omega k^{2} m_{0}^{2}}\left(\frac{n b^{2}}{2 \omega k^{2}}-b d_{1}-c\right)\left(m_{1} y+m_{2}\right) \\
& -\frac{m_{1} n^{2} b^{2}}{\omega^{2} k^{4} m_{0}^{2}}\left(m_{1} y+m_{2}\right) \log \left(m_{1} y+m_{2}\right) \\
& -\frac{1}{2 m_{0}}\left(\left[-m_{0} m_{1} x^{2}+2 d_{0} x\right]\left(m_{1} y+m_{2}\right)^{-2}+\frac{2 n b m_{1}}{\omega k^{2}} x\right) \\
& -\frac{n m_{1}}{\omega k m_{0}^{2}}\left(m_{1} y+m_{2}\right) H(\eta), \\
\eta(x, y)= & m_{0}\left(m_{1} y+m_{2}\right)^{-1} x+d_{1}-\frac{d_{0}}{m_{1}}\left(m_{1} y+m_{2}\right)^{-1}+\frac{n b}{\omega k^{2}} \log \left(m_{1} y+m_{2}\right) .
\end{aligned}
$$


Substituting (5.33) in (5.62), we obtain the following solution of $(2.20)$ :

$$
\begin{aligned}
z(x, y)= & m_{1}\left(\frac{1}{2}-\frac{n A}{\omega k^{2}}\right)\left(m_{1} y+m_{2}\right)^{-1} x^{2} \\
& +\left[-\frac{n m_{1}}{\omega k^{2} m_{0}}\left(b\left(m_{1} y+m_{2}\right)^{-1}+2 d_{1} A+B+\frac{2 n b A}{\omega k^{2}} \log \left(m_{1} y+m_{2}\right)\right)\right. \\
& \left.+\frac{d_{0}^{2}}{m_{1} m_{0}^{2}}\left(\frac{2 n A}{\omega k^{2}}-1\right)\left(m_{1} y+m_{2}\right)^{-1}\right] x \\
& +\frac{n m_{1}}{\omega k^{2} m_{0}^{2}}\left(\frac{n b^{2}}{2 \omega k^{2}}-b d_{1}-c+d_{1}^{2} A+d_{1} B+C\right)\left(m_{1} y+m_{2}\right) \\
& +\frac{b n^{2}}{\omega^{2} k^{4} m_{0}^{2}}\left(2 d_{0} A \log \left(m_{1} y+m_{2}\right)\right. \\
& -m_{1}\left(b+2 d_{1} A+B\right)\left(m_{1} y+m_{2}\right) \log \left(m_{1} y+m_{2}\right) \\
& +\frac{n d_{0}}{\omega k^{2} m_{0}^{2}}\left(2 d_{1} A+B\right)+\lambda_{0} . \\
\omega k^{2} &
\end{aligned}
$$

The function $\phi(y)$ in $(2.20)$ is now given by $\phi(y)=2 m_{1}\left(\omega+k_{0}^{-1} l\right)\left(m_{1} y+m_{2}\right)^{-1}$.

7. $a=b=c=k=0$.

Here, the solution of Eqs. (5.22), (5.24)-(5.26) is found to be

$$
\begin{aligned}
\theta(y) & =m_{0}\left(m_{1} y+m_{2}\right)^{-1}, \quad \beta(y)=d_{1}, \quad \psi(y)=d_{0}\left(m_{1} y+m_{2}\right)^{-1}, \\
\lambda(y) & =\frac{m_{1} d_{0}^{2}}{2 m_{0}^{2}}\left(m_{1} y+m_{2}\right)^{-1},
\end{aligned}
$$

where $m_{0}, m_{1}, m_{2}, d_{0}$, and $d_{1}$ are arbitrary constants. In this case, the similarity form of the solution of (2.20) as obtained from (5.30)-(5.31) and (5.64) is

$$
\begin{aligned}
& z(x, y)=\frac{m_{1}}{2 m_{0}^{2}}\left(m_{0} x+d_{0}\right)^{2}\left(m_{1} y+m_{2}\right)^{-1}+d_{1} H(\eta), \\
& \eta(x, y)=\left(m_{0} x+d_{0}\right)\left(m_{1} y+m_{2}\right)^{-1} .
\end{aligned}
$$

Equations (5.33) and (5.65) give the solution

$$
\begin{aligned}
z(x, y)= & \frac{1}{2}\left(m_{1}-l d_{1} m_{0}^{2}\left(m_{1} y+m_{2}\right)^{-1}\right)\left(m_{1} y+m_{2}\right)^{-1} x^{2} \\
& +\left[\frac{d_{0} m_{1}}{m_{0}}+m_{0} d_{1}\left(-l d_{0}\left(m_{1} y+m_{2}\right)^{-1}+B\right)\right]\left(m_{1} y+m_{2}\right)^{-1} x \\
& +\left[\frac{m_{1} d_{0}^{2}}{2 m_{0}^{2}}+d_{0} d_{1}\left(-\frac{l d_{0}}{2}\left(m_{1} y+m_{2}\right)^{-1}+B\right)\right]\left(m_{1} y+m_{2}\right)^{-1}+C
\end{aligned}
$$


where $B$ and $C$ are arbitrary constants. The function $\phi(y)$ is found to be

$$
\phi(y)=-2 m_{1}(2-\omega)\left(m_{1} y+m_{2}\right)^{-1}-2 \omega d_{1} m_{0}^{2}\left(2 k_{0}+l\right)\left(m_{1} y+m_{2}\right)^{-2} .
$$

The solution (4.44) obtained by the group-theoretic method is easily deduced from (5.66) if we choose $m_{0}=m_{1}=d_{1}=1, l=0$, and $2 B=-d_{0}$ in the latter. In the present case, $F(\eta) \equiv H(\eta)$.

6. Solutions quadratic in $x$. The piston driven flows characterised by the boundary condition (2.32) may be described by the solution

$$
z(x, y)=F_{0}(y)+G(y) x+\frac{1}{2} H(y) x^{2} .
$$

Substituting (6.1) in (2.20) and equating the coefficients of different powers of $x$ to zero, we get the following system of nonlinear ordinary differential equations:

$$
\begin{aligned}
H^{\prime \prime}+[(4-n) H-\phi] H^{\prime}+(2-n) H^{3}-\phi H^{2} & =0 \\
G^{\prime \prime}+[(2-n) H-\phi] G^{\prime}+\left[(2-n) H^{2}-\phi H+2 H^{\prime}\right] G & =0 \\
F_{0}^{\prime \prime}-(n H+\phi) F_{0}^{\prime}-\frac{1}{2}[\phi-(2-n) H] G^{2}+2 G G^{\prime} & =0 .
\end{aligned}
$$

We choose $\phi=4 A_{0} H$, where $A_{0}$ is a constant. We may then solve (6.2) to obtain

$$
H=\left(C_{0}+B_{0} y\right)^{-1}, \quad B_{0}=\omega-2 A_{0},
$$

where $C_{0}$ is a constant. Now, (6.3) is easily solved:

$$
G(y)=D_{0}\left(C_{0}+B_{0} y\right)^{m_{1}}, \quad m_{1}=-1-2 B_{0}^{-1} .
$$

A solution of (6.4) is

$$
F_{0}(y)=\frac{1}{6} D_{0}^{2}\left(C_{0}+B_{0} y\right)^{2 m_{1}+1} .
$$

Thus, the solution of $(2.20)$ in the present case is

$$
z(x, y)=\frac{1}{6} D_{0}^{2}\left(C_{0}+B_{0} y\right)^{2 m_{1}+1}+D_{0}\left(C_{0}+B_{0} y\right)^{m_{1}} x+\frac{1}{2}\left(C_{0}+B_{0} y\right)^{-1} x^{2}
$$

and the sound speed and the density are given by

$$
\begin{aligned}
& a^{2}=\gamma(\gamma-1) a_{0}^{2}\left[\frac{1}{2} D_{0}^{2}\left[1+\frac{1}{3}\left(2 n_{2}+1\right) B_{0}\right]\left(C_{0}+B_{0} y\right)^{2 n_{2}}\right. \\
& \left.\quad+D_{0}\left(n_{2} B_{0}+1\right)\left(C_{0}+B_{0} y\right)^{n_{2}-1} x+\frac{1}{2}\left(1-B_{0}\right)\left(C_{0}+B_{0} y\right)^{-2} x^{2}\right], \\
& \quad \rho=\frac{a^{2 /(\gamma-1)}}{\gamma^{1 /(\gamma-1)}(\gamma-1)^{\gamma /(\gamma-1)}}\left(C_{0}+B_{0} y\right)^{4 \gamma A_{0} /\left((\gamma-1) B_{0}\right)} .
\end{aligned}
$$


7. Solutions by equation-splitting. In this section we obtain two solutions of (2.20) by splitting it into two equations and hence solving them.

1. We split equation (2.20) into

$$
\begin{aligned}
\omega z_{x}^{2}-n z_{y} & =0 \\
z_{y y}+2 z_{x} z_{x y}-\phi(y)\left(z_{y}+\frac{1}{2} z_{x}^{2}\right) & =0 .
\end{aligned}
$$

Manipulating equations (7.1)-(7.2) we get

$$
z_{y y}+2 z_{x} z_{x y}-2 \omega_{1} \phi(y) z_{y}=0, \quad \omega_{1}=1 /(2 \omega) .
$$

We may write (7.3) as

$$
\frac{\partial}{\partial y}\left(z_{x}^{2}-\frac{1}{\omega_{0}} z_{y}\right)=2 \omega_{1} \frac{f^{\prime}}{f} z_{y}-\left(1+\frac{1}{\omega_{0}}\right) z_{y y}
$$

Equations (7.1) and (7.4) require that

$$
2 \omega_{1} f^{-1} f^{\prime} z_{y}-\left(1+1 / \omega_{0}\right) z_{y y}=0 .
$$

Integrating (7.5) twice with respect to $y$, we have

$$
z=v(x) \int^{y} f^{\alpha_{2}}(t) d t+w(x), \quad \alpha_{2}=2 \gamma /(3 \gamma-1)
$$

where $v(x)$ and $w(x)$ are functions of $x$. Substitution of (7.6) in (7.1) leads to the determination of the functions $v(x)$ and $w(x)$ :

$$
v(x)=\left(b_{0} x+b_{2}\right)^{2}, \quad w(x)=b_{0} b_{1} x^{2}+2 b_{1} b_{2} x+b_{3},
$$

where $b_{0}, b_{1}, b_{2}$, and $b_{3}$ are arbitrary constants. The function $f$ is obtained from (7.1), (7.6), and (7.7):

$$
f(y)=\left(\frac{1}{2 b_{1} \sqrt{\omega_{0}}}-2 \omega_{0}^{1 / 2} A y\right)^{(-3 \gamma+1) / \gamma}
$$

where $A$ is a constant. Thus the solution of $(2.20)$ is

$$
z(x, y)=\frac{1}{2 A \sqrt{\omega_{0}}}\left(b_{0} x+b_{2}\right)^{2}\left(\frac{1}{2 b_{1} \sqrt{\omega_{0}}}-2 \omega_{0}^{1 / 2} A y\right)^{-1}+b_{0} b_{1} x^{2}+2 b_{1} b_{2} x+b_{3}
$$

In the present case the equation of state $(2.7)$ is

$$
p=(\gamma-1)^{\gamma} \rho^{\gamma}\left(\frac{1}{2 b_{1} \sqrt{\omega_{0}}}-2 \omega_{0}^{1 / 2} A y\right)^{-3 \gamma+1} .
$$

2. Another solution of $(2.20)$ is obtained if we split it as

$$
n z_{x x}+\phi(y)=0 \quad \text { and } \quad z_{y y}+2 z_{x} z_{x y}-\frac{1}{n} \phi(y) z_{x}^{2}=0 .
$$


Integrating the first of (7.11) twice with respect to $x$ we get

$$
z(x, y)=-\frac{1}{n}\left[\frac{1}{2} \phi(y) x^{2}+V(y) x+W(y)\right]
$$

Substitution of (7.12) in the second of (7.11) leads to the following system of nonlinear ordinary differential equations for $\phi(y), V(y)$, and $W(y)$ :

$$
\begin{aligned}
n^{2} \phi^{\prime \prime}-4 n \phi \phi^{\prime}+2 \phi^{3} & =0, \\
n^{2} V^{\prime \prime}-2 n \frac{d}{d y}\left(\phi V^{\prime}\right)+2 \phi^{2} V & =0, \\
n^{2} W^{\prime \prime}-2 n V V^{\prime}+\phi V^{2} & =0 .
\end{aligned}
$$

The solution of the system $(7.13)-(7.15)$ is

$$
\begin{aligned}
\phi(y) & =-\frac{\gamma-1}{\gamma} \frac{\beta}{\alpha+\beta y}, \\
V(y) & =b_{4}(\alpha+\beta y)^{-1}+b_{5}, \\
W(y) & =\frac{\gamma}{(\gamma-1)}\left[\left(b_{6} y+b_{7}\right)+\frac{b_{5}^{2}}{\beta}(\alpha+\beta y)[-1+\log (\alpha+\beta y)]\right. \\
& \left.-\frac{1}{2 \beta} b_{4}^{2}(\alpha+\beta y)^{-1}\right]
\end{aligned}
$$

where $b_{4}, b_{5}, b_{6}$, and $b_{7}$ are free constants.

Thus the solution of $(2.20)$ in the present case is

$$
\begin{aligned}
z(x, y)=-\frac{1}{n}[ & -\frac{\gamma-1}{2 \gamma} \frac{\beta}{\alpha+\beta y} x^{2}+\left(b_{4}(\alpha+\beta y)^{-1}+b_{5}\right) x \\
& +\frac{\gamma}{(\gamma-1)}\left[\left(b_{6} y+b_{7}\right)+\frac{b_{5}^{2}}{\beta}(\alpha+\beta y)[-1+\log (\alpha+\beta y)]\right.
\end{aligned}
$$

In this case, the equation of state (2.7) becomes

$$
p=(\gamma-1)^{\gamma}(\alpha+\beta y)^{1-\gamma} \rho^{\gamma}
$$

8. Results and Conclusions. Equation (2.20) derived in Sec. 2 from the conservation form of the one-dimensional gas dynamic equations (2.1)-(2.3) does not seem to have attracted much attention. This complicated partial differential equation, with several linear and nonlinear terms and with a function $\phi(y)$ of the independent variable multiplying two of them, does not appear to possess any symmetries. Yet, the details of Secs. 2-7 show that it allows considerable analysis, symmetries, and several families of 
solutions. It also has first integrals (3.11) which allow a generalization (3.12) of the Riemann invariants. One of the first integrals is then treated as first-order partial differential equation, yielding the solutions (3.17) and (3.22).

Equation (2.20) is a "good" test equation to compare which of the two approaches exploiting symmetries - the classical Lie group method and the direct similarity approach proposed by Clarkson and Kruskal [17] — leads to more general similarity solutions. The similarity form (5.30)-(5.31) of the solution of (2.20), obtained by the direct similarity method, seems to represent a much larger family of solutions. The similarity function $H(\eta)$, appearing in the form (5.30)-(5.31) of the solution, is given by the nonlinear ordinary differential equation (5.32) while the other functions involved in the similarity form, namely, $\theta(y), \beta(y), \psi(y)$, and $\lambda(y)$ may be found from the determinate set of nonlinear coupled ordinary differential equations (5.22), (5.24)-(5.26).

Even though it does not seem possible to analytically solve the determinate system (5.22), (5.24)-(5.26), and (5.32), it may be solved numerically, if suitable initial conditions, pertinent to them, can be identified. The generality of this system is confirmed by the fact that all the solutions obtained through the classical Lie group method can be recovered as special cases of this system. The solutions obtained by the two methods appear distinct: each of the ordinary differential equations (4.22), (4.32), and (4.39) when compared with Eq. (5.32) obtained by the direct similarity method and governing the similarity functions, appear different, but, as we have shown in Sec. 5, a simple change of variables (see (5.44), (5.53), and (5.57)), reconciles these apparently distinct equations.

There are five distinct classes of solutions obtained by using the direct similarity approach. For all five classes, the solutions have the form

$$
z(x, y)=Q_{0}(y)+Q_{1}(y) x+Q_{2}(y) x^{2},
$$

with $Q_{2}(y)=\sigma\left(m_{1} y+m_{2}\right)^{-1}$, where $\sigma, m_{1}$, and $m_{2}$ are arbitrary constants. The functions $Q_{1}(y)$ and $Q_{0}(y)$ have the following five distinct forms:

Class (1):

$$
\begin{aligned}
& Q_{1}(y)=\frac{n}{k \omega}\left(1+2 n_{1}\right) m_{0}^{2}\left[-\frac{b}{a} A+B\right]\left(m_{1} y+m_{2}\right)^{-n_{1}-1} \\
& Q_{0}(y)=\left[\frac{b^{2} A}{4 a^{2}}-\frac{b B}{a}+C-\frac{b^{2}-4 a c}{4 a k}\right] \frac{n}{k \omega}\left(1+2 n_{1}\right) m_{0}^{2} m_{1}\left(m_{1} y+m_{2}\right)^{-2 n_{1}-1} .
\end{aligned}
$$

Class (2):

$$
\begin{aligned}
& Q_{1}(y)=\frac{m_{1}}{m_{0}}\left[d_{0}-\frac{2 n}{\omega k}\left(2 d_{0} A+B e^{m_{1} y}\right)\right] \\
& Q_{0}(y)=\lambda_{0}-\frac{2 n m_{1} d_{0}^{2} A}{\omega k m_{0}^{2}}-\frac{d_{0}^{2} m_{1}^{2}}{2 m_{0}^{2}}\left(1+\frac{8 a n}{\omega k^{2}}\right) y-\frac{2 n m_{1} d_{0}}{\omega k m_{0}^{2}}\left(B+C e^{m_{1} y}\right) e^{m_{1} y} .
\end{aligned}
$$


Class (3):

$$
\begin{aligned}
Q_{1}(y)= & {\left[-\frac{n m_{1}}{\omega k^{2} m_{0}}\left(b\left(m_{1} y+m_{2}\right)^{-1}+2 d_{1} A+B+\frac{2 n b A}{\omega k^{2}} \log \left(m_{1} y+m_{2}\right)\right)\right.} \\
Q_{0}(y)= & \frac{d_{0}^{2}}{m_{1} m_{0}^{2}}\left(\frac{1}{2}-\frac{d_{0}}{m_{0}}\left(\frac{2 n A}{\omega k^{2}}-1\right)\left(m_{1} y+m_{2}\right)^{-1}\right], \\
& +\frac{n m_{1}}{\omega k^{2} m_{0}^{2}}\left(\frac{n b^{2}}{2 \omega k^{2}}-b d_{1}-c+d_{1}^{2} A+d_{1} B+C\right)\left(m_{1} y+m_{2}\right) \\
& +\frac{b n^{2}}{\omega^{2} k^{4} m_{0}^{2}}\left(2 d_{0} A \log \left(m_{1} y+m_{2}\right)-m_{1}\left(b+2 d_{1} A+B\right)\left(m_{1} y+m_{2}\right) \log \left(m_{1} y+m_{2}\right)\right. \\
& \left.-\frac{b n m_{1} A}{\omega k^{2}}\left(m_{1} y+m_{2}\right)\left[\log \left(m_{1} y+m_{2}\right)\right]^{2}\right)+\frac{n d_{0}}{\omega k^{2} m_{0}^{2}}\left(2 d_{1} A+B\right)+\lambda_{0} .
\end{aligned}
$$

Class (4):

$$
\begin{aligned}
& Q_{1}(y)=\left[\frac{n m_{1}}{\omega k m_{0}}\left(\frac{b}{k}+B-\frac{2 b n A}{\omega k^{2}} \log \left(m_{1} y+m_{2}\right)+2 A\left(d_{0} y+d_{1}\right)\right)\right. \\
& \left.-\frac{d_{0}}{m_{0}}\left(m_{1} y+m_{2}\right)\right]\left(m_{1} y+m_{2}\right)^{-1} \\
& Q_{0}(y)=\frac{n m_{1}}{\omega k m_{0}^{2}}\left[\frac{1}{k}\left(c-\frac{n b^{2}}{2 \omega k^{2}}\right)+\frac{b}{k}\left(d_{0} y+d_{1}\right)\right. \\
& -\frac{n b^{2}}{\omega k^{3} m 62_{0}} \log \left(m_{1} y+m_{2}\right)+\frac{n^{2} b^{2} A}{\omega^{2} k^{4}}\left[\log \left(m_{1} y+m_{2}\right)\right]^{2} \\
& +A\left(d_{0} y+d_{1}\right)^{2}-\frac{2 n b A}{\omega k^{2}}\left(d_{0} y+d_{1}\right) \log \left(m_{1} y+m_{2}\right) \\
& \left.-\frac{n b B}{\omega k^{3}} \log \left(m_{1} y+m_{2}\right)+B\left(d_{0} y+d_{1}\right)+C\right]\left(m_{1} y+m_{2}\right)^{-1} \\
& -\frac{d_{0}^{2}}{2 m_{0}^{2}} y+\lambda_{0}
\end{aligned}
$$

Class (5):

$$
\begin{aligned}
& Q_{1}(y)=\left[\frac{d_{0} m_{1}}{m_{0}}+m_{0} d_{1}\left(-l d_{0}\left(m_{1} y+m_{2}\right)^{-1}+B\right)\right]\left(m_{1} y+m_{2}\right)^{-1}, \\
& Q_{0}(y)=\left[\frac{m_{1} d_{0}^{2}}{2 m_{0}^{2}}+d_{0} d_{1}\left(-\frac{l d_{0}}{2}\left(m_{1} y+m_{2}\right)^{-1}+B\right)\right]\left(m_{1} y+m_{2}\right)^{-1}+C .
\end{aligned}
$$

Other approaches - solutions from the first integrals and the assumed quadratic (in $x$ ) form of the solution in Sec. 6-do not lead to any new solutions; these are, in fact, special cases of the general form (5.30)-(5.31) obtained from the direct similarity approach. The only exception is the adhoc approach which splits Eq. (2.20): it leads to one new solution, namely (7.17), not obtained from any other approach. 
Referring now to the boundary conditions for these solutions, arising from the physical problem, namely, (2.29) $-(2.30)$ at the shock curve $y=y_{0}(x)$, and (2.32) at the piston $y=0$, we have given a few illustrative examples, (see (4.29), (4.34), and (4.41) with $a_{3}=a_{5}=a_{8}=0$ ). The solutions (4.29) and (4.41) with $a_{3}=a_{5}=a_{8}=0$, satisfy the condition (2.33) for a strong shock while the solution (4.34) satisfies the conditions (2.29)(2.30) for a shock of finite strength. It may be possible to satisfy boundary conditions at the shock for other classes of solutions that we have found. More general solutions with strong shocks can be obtained by solving the ordinary differential equations such as (4.22), (4.32), and (5.32)) subject to initial conditions. For example, for Eq. (4.22), we find that $F(0)=0$ and $F^{\prime}(0)=0$ so that a numerical solution of (4.22) may be found using these initial conditions.

We conclude that the nonlinear partial differential equation (2.20) arising from the conservation form of one-dimensional gas-dynamic equations displays many symmetries and possesses a great variety of solutions. Other partial differential equations for "potentiallike" functions (see Steketee [8] and Ardavan-Rhad [10]) arising from one-dimensional gas-dynamic equations may be treated by the methods expounded in the present paper.

\section{REFERENCES}

[1] R. Courant and K. O. Friedrichs, Supersonic Flow and Shock Waves, Interscience, New York, 1948

[2] L. I. Sedov, Similarity and Dimensional Methods in Mechanics, Academic Press, New York, 1959

[3] J. B. Keller, Spherical, cylindrical and one-dimensional gas flows, Quart. Appl. Math. 14, 171-184 (1956)

[4] G. C. McVittie, Spherically symmetric solutions of the equations of gas dynamics, Proc. Roy. Soc. London Ser. A 220, 339-355 (1953)

[5] M. H. Martin, The propagation of a plane shock into a quiet atmosphere, Canadian Journal of Mathematics 5, 37-39 (1953)

[6] G. S. S. Ludford and M. H. Martin, One dimensional anisentropic flows, Comm. Pure Appl. Math. 7, 45-63 (1954)

[7] J. A. Steketee, Unsteady rectilinear flows of a non-homentropic gas, Acta Astronaut. 6, 413-434 (1979)

[8] J. A. Steketee, Transformations of the equations of motion for the unsteady rectilinear flow of a perfect gas, J. Engrg. Math. 10, 69-94 (1976)

[9] P. Smith, Anisentropic rectilinear gas flows, Appl. Sci. Res. A12, 66-72 (1964)

[10] H. Ardavan-Rhad, The decay of a plane shock wave, J. Fluid Mech. 43, 737-751 (1970)

[11] M. D. Ustinov, Ideal gas flow behind a finite-amplitude shock wave, Izv. Akad. Nauk. SSSR, Mekh. Zhid. Gaza. 2, 88-90 (1967)

[12] P. L. Sachdev and A. Venkataswamy Reddy, Some exact solutions describing unsteady plane gas flows with shocks, Quart. Appl. Math. 40, 249-272 (1982)

[13] P. L. Sachdev, Neelam Gupta, and D. S. Ahluwalia, Exact analytic solutions describing unsteady plane gas flows with shocks of arbitrary strength, Quart. Appl. Math. 50, 677-726 (1992)

[14] M. D. Ustinov, Approximate solution to nonself-similar problem of motion of a piston after an impact, Izv. Akad. Nauk. SSSR, Mekh. Zhid. Gaza. 6, 167-171 (1982)

[15] M. D. Ustinov, Motion of a piston under the influence of gas pressure in the presence of an initial temperature gradient, Izv. Akad. Nauk. SSSR, Mekh. Zhid. Gaza. 2, 177-180 (1984)

[16] M. D. Ustinov, Some one-dimensional unsteady adiabatic gas flows with plane symmetry, Izv. Akad. Nauk. SSSR, Mekh. Zhid. Gaza. 5, 96-104 (1986)

[17] P. Clarkson and M. D. Kruskal, New similarity reductions of the Boussinesq equation, J. Math. Phys. 30, 2201-2213 (1989)

[18] G. W. Bluman and J. D. Cole, The general similarity solution of the heat equation 18, 1025-1042 (1969) 